\title{
A Four-Scale Bidirectional Reflectance Model Based on Canopy Architecture
}

\author{
Jing M. Chen and Sylvain G. Leblanc
}

\begin{abstract}
Open boreal forests present a challenge in understanding remote sensing signals acquired with various solar and view geometries. Much research is needed to improve our ability to model the bidirectional reflectance distribution (BRD) for retrieving the surface information using measurements at a few angles. The geometric-optical bidirectional reflectance model presented in this paper considers four scales of canopy architecture: tree groups, tree crowns, branches and shoots. It differs from the Li-Strahler's model in the following respects: 1) the assumption of random spatial distribution of trees is replaced by the Neyman distribution which is able to model the patchiness or clumpiness of a forest stand; 2) the multiple mutual shadowing effect between tree crowns is considered using a negative binomial and the Neyman distribution theory; 3 ) the effect of the sunlit background is modeled using a canopy gap size distribution function that affects the magnitude and width of the hotspot; 4) the branch architecture affecting the directional reflectance is simulated using a simple angular radiation penetration function; and 5) the tree crown surface is treated as a complex surface with microscale structures which themselves generate mutual shadows and a hotspot. All these scales of canopy architecture are shown to have effects on the directional distribution of the reflected radiance from conifer forests. The model results compare well with a data set from a boreal spruce forest.
\end{abstract}

\section{NOMENCLATURE}

$A \quad$ Quadrat size.

$A_{S} \quad$ Averge shoot projected area.

$B \quad$ Domain size (pixel size).

$C(\theta) \quad G(\theta) / \sin (\theta)$.

$D \quad$ Number of trees in the domain $B$.

$F_{a s}(\lambda) \quad$ Accumulated gap size distribution inside tree crowns.

$F_{a t}(\lambda) \quad$ Accumulated gap size distribution between tree crowns.

$G(\theta) \quad$ Projection of unit leaf area.

$H$ Effective height $\left(H_{a}+H_{b}+\frac{1}{3} H_{c}\right) / \cos \left(\theta_{s}\right)$.

$H_{a}$ Height of the lower part of the tree (trunk space).

$H_{b}$ Height of cylinders.

$H_{c} \quad$ Height of cones.

$H_{t}$ Total height of the tree crown $\left(H_{c}+H_{b}\right)$.

$L$ Leaf area index (LAI).

$L_{H}$

LAI accumulated horizontally $(L \cdot \bar{s}(\theta=$ $\left.90^{\circ}\right)$ ).

Manuscript received March 27, 1996; revised February 28, 1997.

J. M. Chen is with the Canada Centre for Remote Sensing, Ottawa, Ont., Canada K1A 0Y7 (e-mail: chen@ccrs.nrcan.gc.ca).

S. G. Leblanc is with Intermap Technologies Ltd., Nepean, Ont., Canada K2E 1A2

Publisher Item Identifier S 0196-2892(97)05511-3.
$L_{\circ}$

$L_{s}$

$L_{t}$

$m$

$m_{1}$

$m_{2}$

$n$

$P_{G}$

$P_{\text {gap }}(\theta)$

$P_{i g}$

$P_{s}$

$P_{v g}$

$P_{v g-r}$

$P_{v g-c}$

$P_{s t}$

$P_{T}$

$P_{t i}$

$P_{v}$

$P(x)$

$P_{N}(i)$

$P_{s}(\lambda)$

$P_{t}(\lambda)$

$Q_{1 \text { tot }}, Q_{2 \text { tot }}$

$r$

$\bar{s}(\theta)$

$R_{G}$

$R_{T}$

$R_{Z G}$

$R_{Z T}$

$R$

$S_{g}$

$S_{g c}$

$S_{g b}$

$t_{i c} t_{i b}$
Mean LAI accumulated over the view or sun path within one tree crown.

Clumping-adjusted projected tree crown element area index.

Clumping-adjusted projected tree crown area index.

Mean number of trees in a quadrat.

Mean number of cluster per quadrat.

Cluster mean size.

Number of quadrats in the domain $B$.

Probability of seeing illuminated ground area. Gap Probability within a tree at the angle $\theta$. Probability of having sunlit ground area.

Total shadowing effect.

Probability of seeing the ground (including clusters and overlap).

Probability of seeing the ground (random tree distribution).

Probability of seeing the ground (clustered tree, without overlap).

Probability of seeing tree crown area $(1-$ $P_{v g}$ ).

Probability of seeing sunlit trees.

Proportion of tree crown surface viewed that is illuminated.

Total view overlapping effect.

Poisson distribution.

Neyman distribution.

Probability of having a gap of size $\lambda$ in a crown.

Probability of having a tree gap of size $\lambda$ between trees.

Probability of seeing sunlit shoots inside the tree crowns (no hotspot consideration).

Radius of the tree crowns.

Mean path length within a crown.

Ground reflectivity.

Tree reflectivity.

Shaded ground surface reflectivity.

Shaded tree surface reflectivity.

Total reflectance.

Shadowed area on the ground produced by one tree.

Shadowed area on the ground produced by the cone part of the tree.

Shadowed area on the ground produced by the cylinder (base) part of the tree.

Tree illuminated surface visible to the viewer. 


\begin{tabular}{|c|c|}
\hline $\begin{array}{l}t_{a c} t_{a b} \\
V\end{array}$ & $\begin{array}{l}\text { Tree crown surface visible to the viewer. } \\
\text { Volume of a tree. }\end{array}$ \\
\hline$V_{g}$ & $\begin{array}{l}\text { Ground surface not seen by viewer because } \\
\text { of one tree. }\end{array}$ \\
\hline$V_{g c}$ & $\begin{array}{l}\text { Ground surface not seen by viewer because } \\
\text { of the cone part of one tree. }\end{array}$ \\
\hline$W_{s}$ & $\begin{array}{l}\text { Mean width of element shadows cast inside } \\
\text { tree crowns. }\end{array}$ \\
\hline$W_{t}$ & $\begin{array}{l}\text { Characteristic mean width of tree crowns pro- } \\
\text { jected to the ground. }\end{array}$ \\
\hline$\alpha$ & Half apex angle. \\
\hline$\gamma_{s}, \gamma_{v}$ & $\begin{array}{l}\text { Angle related to the self-shadowing of the } \\
\text { cone. }\end{array}$ \\
\hline$\gamma_{E}$ & Needle-to-shoot area ratio. \\
\hline$\Gamma(\xi)$ & $\begin{array}{l}\text { First-order scattering (geometric shadow) } \\
\text { phase function of the foliage. }\end{array}$ \\
\hline$\mu$ & Crown foliage density. \\
\hline$\Omega_{E}$ & Clumping index for shoots. \\
\hline$\Omega_{t}$ & Clumping index for trees. \\
\hline$\lambda$ & Gap size. \\
\hline$\lambda_{\min }$ & $\begin{array}{l}\text { Minimum gap size for having an illuminated } \\
\text { surface. }\end{array}$ \\
\hline$\phi$ & $\begin{array}{l}\text { Relative azimuth angle between the sun and } \\
\text { the viewer. }\end{array}$ \\
\hline$\theta_{s}$ & Solar zenith angle (SZA). \\
\hline$\theta_{v}$ & View zenith angle (VZA). \\
\hline$\xi$ & $\begin{array}{l}\text { Angle difference between the sun and the } \\
\text { viewer (phase angle). }\end{array}$ \\
\hline
\end{tabular}

\section{INTRODUCTION}

Q OLAR radiance reflected from the earth's surface is strongly anisotropic and depends on both the sun and observation directions. Such bidirectional reflection behavior has been extensively investigated over various surfaces with remote sensing data (Cihlar et al. [8] and $\mathrm{Wu}$ et al. [37]), ground-based measurements (Deering et al. [10]) and numerical models (see Myneni and Ross [26]). In bidirectional reflectance distribution (BRD) models, vegetated surfaces are often described as turbid media (Gao [12], Jupp and Strahler [17], Otterman and Brakke [30], Verhoef [34], Verstraete [35]) that represent well agricultural crops and grassland. For forests, geometric-optical models ( $\mathrm{Li}$ and Strahler [21], [22], Strahler and Jupp [33]) and hybrid-models (Li et al. [23], Nilson and Peterson [29]) combining geometrical and turbid media have been used. One common challenge for canopy models of all types is the difficulty in simulating the multiple scattering processes within the canopy. Much attention has been given to this problem. The other major challenge is the effect of plant canopy architecture on radiative transfer processes. This challenge has not yet been rigorously tackled in the literature although there have also been quite a few publications on this problem (Borel et al. [1], Goel et al. [15], Myneni and Ross [26]) in addition to geometric-optical models. The meaning of architecture here goes beyond the usual angle distribution of leaves. It also includes the spatial distribution patterns of leaves and higher level structures such as shoots, branches and trees. Architecture at all levels in a plant canopy affects not only the transmission of the solar beam through the canopy, but also the multiple scattering processes contributing to the observed radiances. In forest stands, for example, the number of multiple scattering events between tree crowns is undoubtedly much smaller than between leaves, and the modeling methodology would be exceedingly complex and depends on the treatment of the canopy architecture. In the modeling effort presented here, the attention is first given to a detailed mathematical descriptions of the canopy architecture at various levels.

In Li and Strahler's BRD models [22], a forest stand is assumed to consist of randomly distributed objects containing leaves as turbid media. These two-scale models mark a major advancement in simulating radiation regimes in forest stands as compared with the one-scale turbid-media models, but also dramatically increase the complexity of the models. However, two-scale models may be regarded as much simplified mathematical descriptions of the physical reality. First, trees are generally not randomly distributed in space but are usually clustered at large scales due to variations in the soil and topographic conditions, creating patchiness of forest stands. They are also not randomly positioned when close to each other because of the natural repulsion effect in competition for resources (Franklin et al. [11]). Second, leaves are not randomly distributed within tree crowns. In conifer stands, for example, needles are grouped into shoots, branches and whirls, and all these sub-canopy structures are important in determining the radiation regime, especially the directional reflectance. In the present paper, these two additional scales of plant canopy architecture are considered. Fig. 1 shows the concept of the different radiation transfer models based on canopy architecture at several scales. The objective of this paper is to present the first four-scale BRD model for the purpose of investigating the effect of the different architectural scales on the directional reflection behavior of plant canopies.

\section{MODEL DESCRIPTION}

A BRD model computes the percentage of incident radiation that is reflected by a forest in various directions. It is the proportions of the sunlit and shadowed areas of foliage and ground that are used to determine the BRD. Our model contains several "modules," each with specific tasks. The first module constructs the forest. It includes the spatial distribution of trees within a modeling domain and the macroscale geometry of the trees. By considering the tree crown geometry, the shadowed and illuminated areas of an isolated tree are calculated, and by combining the geometry and tree distribution, the gap fraction and gap size distribution of the canopy are computed as functions of solar or view zenith angle. The gap size distribution is then used to model the hotspot effect. The hotspot occurs on both the tree crowns and the ground. The mathematical treatments on the canopy architecture are presented below.

\section{A. Tree Distribution}

BRD models based on discrete canopy structures usually assume that trees are randomly distributed within a spatial 


\section{Radiation Models}

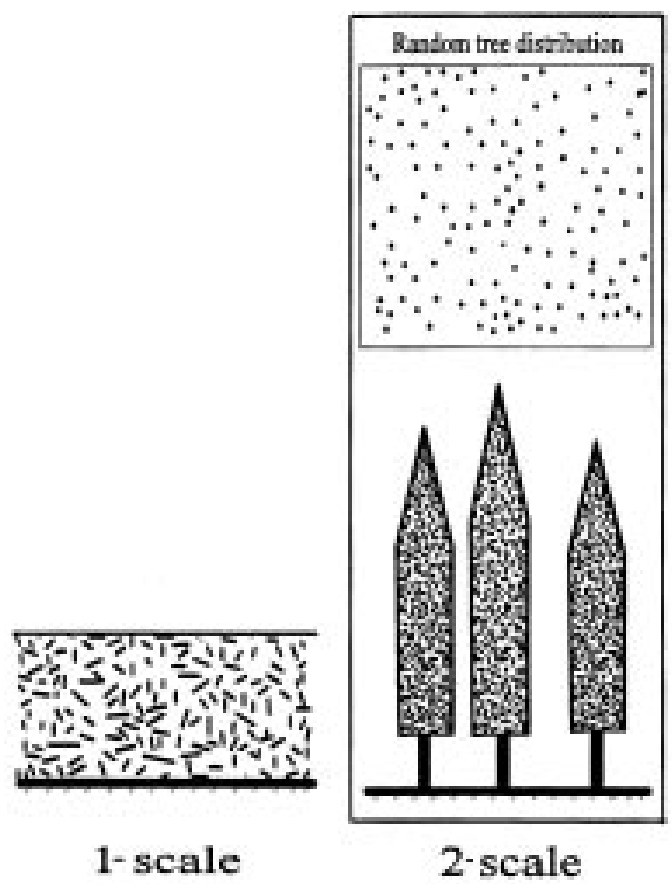

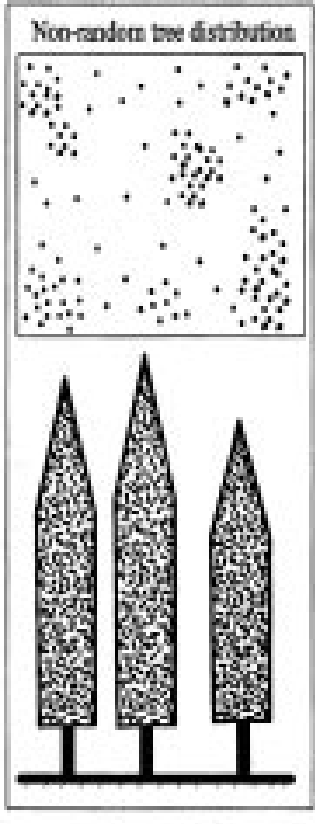

3-scale

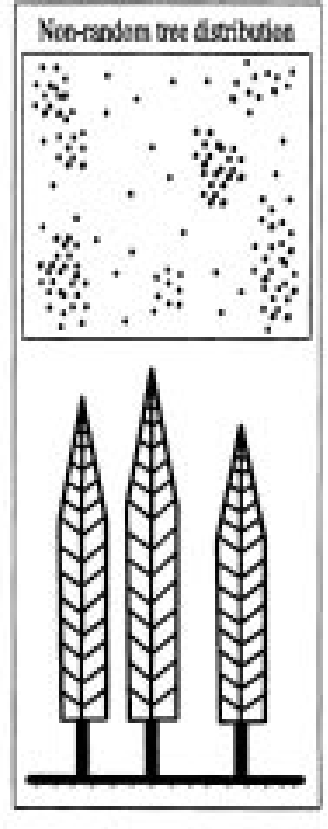

4-scale

Fig. 1. Scales of plant canopy architecture considered in reflectance models: one-scale: turbid media; two-scale: randomly-distributed discrete objects containing turbid media; three-scale: nonrandom discrete objects containing turbid media; and four-scale: nonrandom discrete objects with internal structures (such as branches and shoots).

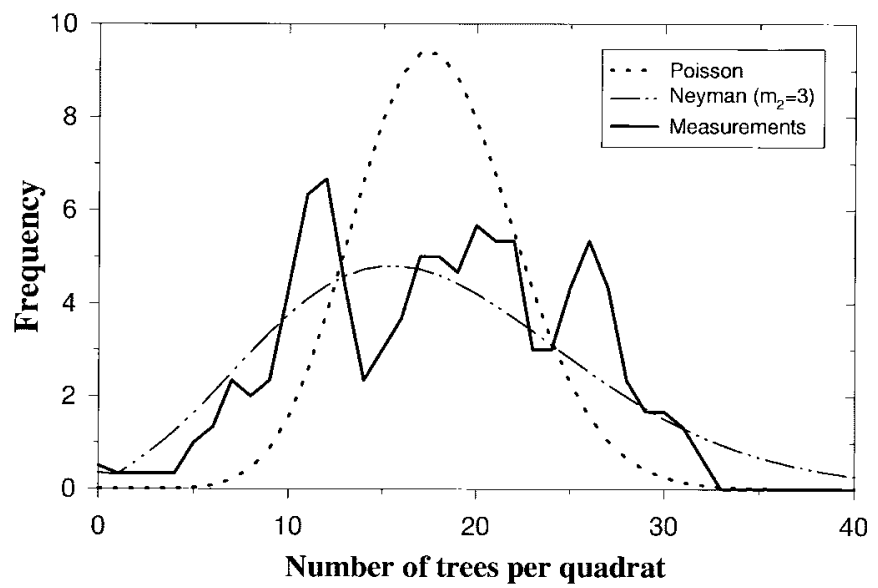

Fig. 2. The tree distribution in a $100 \times 100 \mathrm{~m}^{2}$ boreal jack pine forest divided in 100 quadrats compared with Poisson and Neyman distributions.

domain. This distribution is described using the Poisson theory

$$
P(x)=\frac{e^{-m} m^{x}}{x !}
$$

where $m$ is the average value of the number of objects (in our case, trees) in a sub-domain called quadrat, and $P(x)$ is the probability of finding $x$ objects in the sub-domain. However, in reality, trees are generally not randomly distributed but rather grouped together in various ways. Measurements from a boreal jack pine stand (Fig. 2) show an obvious deviation of the tree distribution from Poisson's random case, i.e., the number of quadrats having a certain number of trees is distributed narrowly around the mean number of trees per quadrat while the measured distribution is much broader. The Poisson tree distribution is in fact an averaging process: as the quadrat size (or the mean number of trees per quadrat) increases trees become more evenly distributed among the various quadrats. The application of this process to represent reality requires a forest stand to be uniform at both small and large scales. However, variations in environmental conditions such as topography, soil and sucessional processes often make trees irregularly distributed, forming patches at various scales. The simple Poisson theory is incapable of describing such patchiness. Nilson and Peterson [29] made a correction to the Poisson theory to model nonrandomness of tree distribution. Neyman [27] (with application to geography by Getis and Boot [13]) developed a method for describing the contagious distribution of larvae. This method called Neyman type A has been used by Franklin et al. [11] for the investigation of tree distributions. It assumes that trees are first combined in groups and the spatial distribution of the centre of a group follows the Poisson process. The mean size of the groups is a required model input depending on the degree of clumping of trees. Centred around a given mean group size, there are also probabilities for other group sizes determined again by the Poisson theory. Hence, the Neyman type A distribution can also be called the double-Poisson distribution. For the convenience of mathematical description, a group must fall completely into a quadrat with the centre of the group. The size of the quadrat denoted by $A$, used in the model, should represent the extent of radiation interaction between trees, i.e., the horizontally-projected path length of a solar beam through 


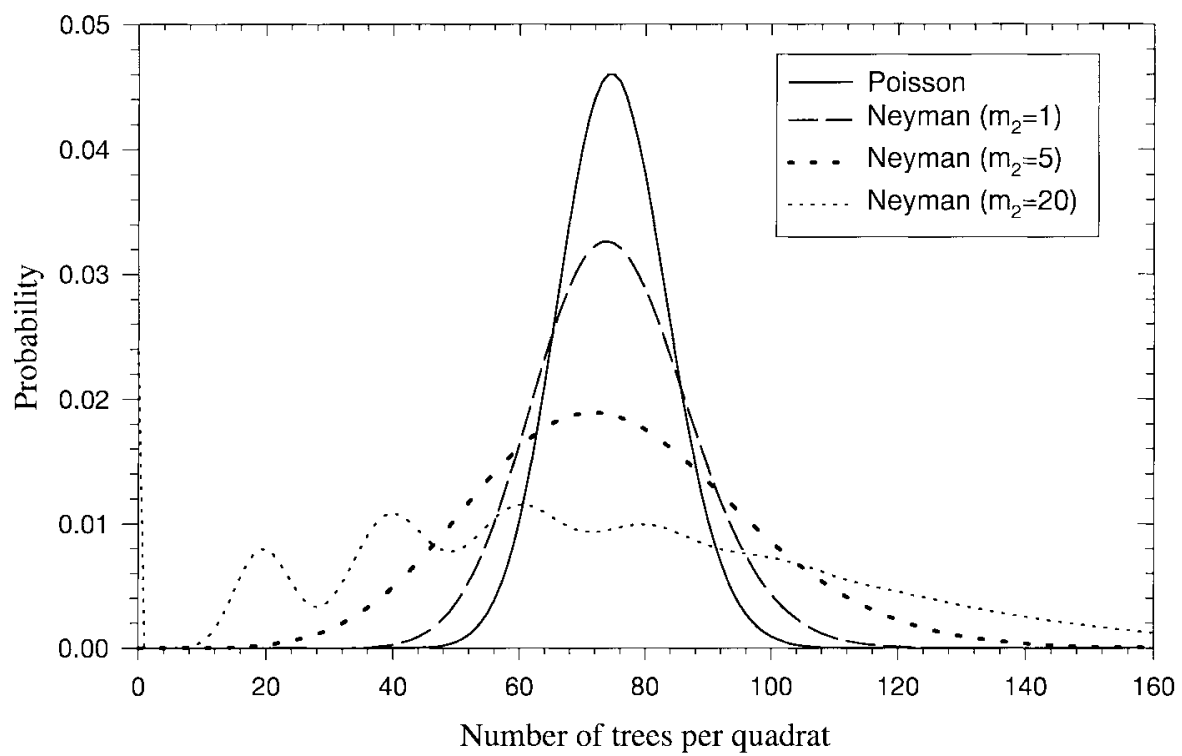

Fig. 3. Comparison between the Poisson and the Neyman distributions of various group sizes $\left(m_{2}\right)$ with an average of 75 trees per quadrat.

the canopy. The modeling domain is equivalent to the size of a pixel in remote sensing. In selecting the quadrat size there is also a computational constraint: the larger the group, the larger should be the quadrat. For a $100 \times 100 \mathrm{~m}^{2}$ domain with 3000 trees, for example, it is preferable to divide the domain into at least ten quadrats to avoid overly populated quadrats that could be difficult to handle numerically.

According to the conditional probability theory, the probability of having $i$ trees given $j$ groups in a quadrat, $P(i \mid j)$, times the probability of having $j$ groups in the quadrat, $P(j)$, gives the probability of having $i$ trees in the quadrat given $j$ groups. The probability of having $i$ trees in the quadrat, $P(i)$, is the summation of all the conditional probabilities for $j$ :

$$
P(i)=\sum_{j}^{\infty} P(i \mid j) P(j) .
$$

When both $P(j)$ and $P(i \mid j)$ are determined by the Poisson process, we obtain

$$
\begin{gathered}
P_{N}\left(i ; m_{1} ; m_{2}\right)=e^{-m_{1}} \frac{m_{2}^{i}}{i !} \sum_{j=1}^{\infty} \frac{\left[m_{1} e^{-m_{2}}\right]^{j}}{j !} \cdot j^{i} \\
\text { for } i=0,1,2, \cdots
\end{gathered}
$$

where

$$
m_{1}=\frac{m^{2}}{\nu-m}
$$

is the mean number of groups per quadrat and

$$
m_{2}=\frac{\nu-m}{m}
$$

is the cluster mean size. $m=m_{1} m_{2}$ is the mean and $\nu=m_{1} m_{2}\left[1+m_{2}\right]$ is the variance of the distribution of the number of trees per quadrat and can be measured in a real forest stand. In creating this distribution, the following implicit assumptions are used [13].

1) Within the modeling domain each quadrat is equally likely to receive a cluster and the placement of a cluster is independent of the placement of any other clusters.

2) The parameter $m_{1}$ corresponds to a priori value of the density of a cluster and $m_{2}$ corresponds to a priori value of the mean size of clusters (optional).

3) In conformity with a Poisson model the variance about the mean size of cluster is equal to the mean size of cluster.

4) The assignment of a cluster size to one location is independent of the assignment of any other cluster size or cluster location (optional).

5) The points in a cluster are propagated by a "progenitor" who is located at the site of each cluster (pseudocontagious assumption).

If the real distribution is not known, $m_{2}$ will be fixed at a low value $\left(m_{2}=1\right.$ implies a tree distribution close to the random case). Comparison with field data will later determine the values for those parameters.

Fig. 3 shows a random tree distribution (Poisson) in comparison with Neyman distributions with the mean grouping $m_{2}$ of one, five, and 20 trees per group. In the calculation, the average density was 75 trees per quadrat (1500 trees/ha with 20 quadrats of $500 \mathrm{~m}^{2}$ each). Groupings of 1 and 5 produce distributions centred at 75 and increase the standard deviation from the random case. $m_{2}=1$ means that the grouping is mostly random (group of one) but it contains probabilities of having groups of two trees or more and therefore has a broader distribution than the random case. If the group size is too large compared to the overall mean $(m)$, the Neyman distribution becomes variable with peaks at multiples of that size. As a result, the curve for $m_{2}=20$ in Fig. 3 shows maxima at 20, 40 , and 60 trees per quadrat. At $i=0$, the number of quadrats (having no trees) increases with group size, suggesting that for larger group sizes, the probability of having empty quadrats 


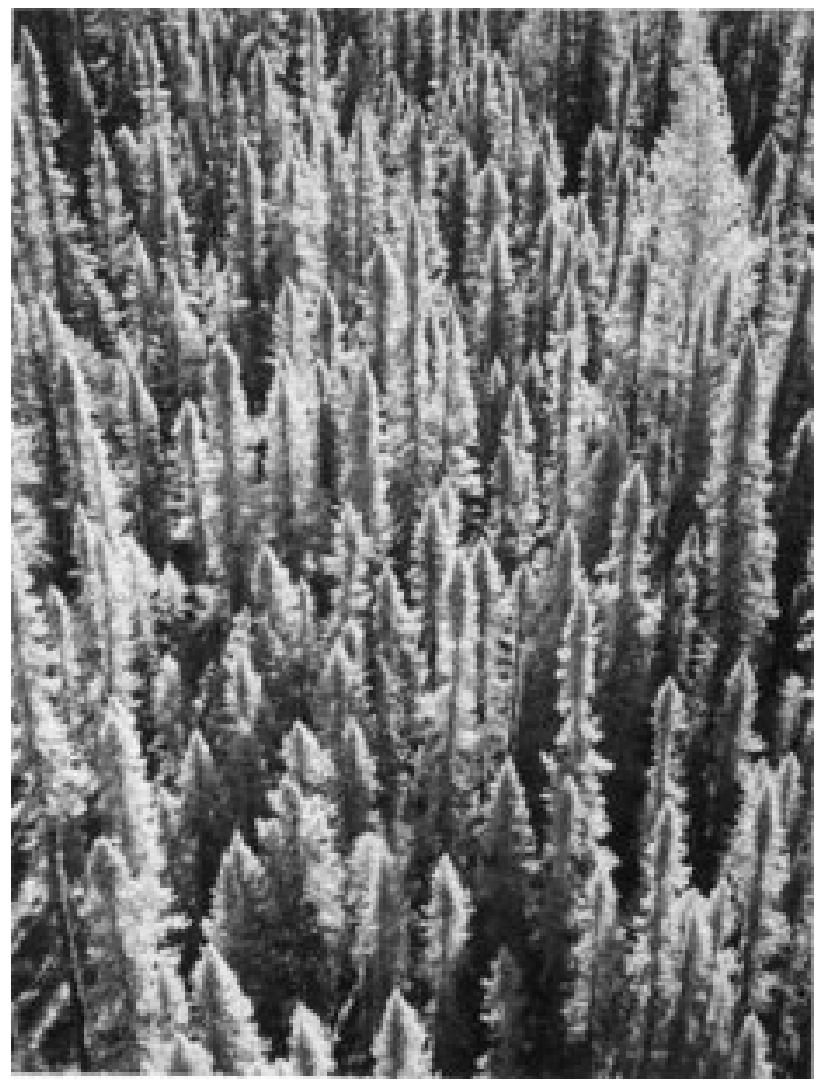

Fig. 4. A photograph of an old black spruce forest, near Candle Lake, Saskatchewan, investigated in this paper.

is greater. In Fig. 2, measurements from a jack pine forest are compared with the Poisson and Neyman distributions. The domain studied was one hectare with 1793 trees divided into 100 quadrats. A Neyman distribution with grouping of three simulates the measurements more closely than the Poisson theory. Processes other than Neyman could be used in this kind of analysis. For its simplicity and for the first analysis of tree clumping in forest canopies, only the Neyman, i.e., the double-Poisson process is used in this paper.

\section{B. Tree Crown Projection}

Conifer tree crowns have been modeled with a cone shape by $\mathrm{Li}$ and Strahler [19]. Nilson and Peterson [29] added a cylinder below the cone in their simulations. A photograph taken in a mature black spruce stand near Candle Lake, Saskatchewan, Canada (Fig. 4), shows that the trees essentially have a cylindrical shape with a conical top. In our model, a tree crown is then assumed to consist of a cone and a cylinder. In common with Li and Strahler's [19] model, the conical part is described with two factors: its radius $(r)$ and its half apex angle $(\alpha)$. In this paper, all trees have the same half apex angle $\left(\alpha=13^{\circ}\right)$. Fig. 5 shows how a tree crown is modeled. $H_{b}$ is the height of the cylinder. The height of the cone is defined by its apex angle and the radius of the tree: $H_{c}=r / \tan (\alpha)$. The total height of the tree crown is $H_{t}=H_{b}+H_{c}$.

A cone casts a shadow on the ground in addition to the cylinder beneath it only if the solar zenith angle is larger than one-half of the apex angle. This shadow area is expressed as

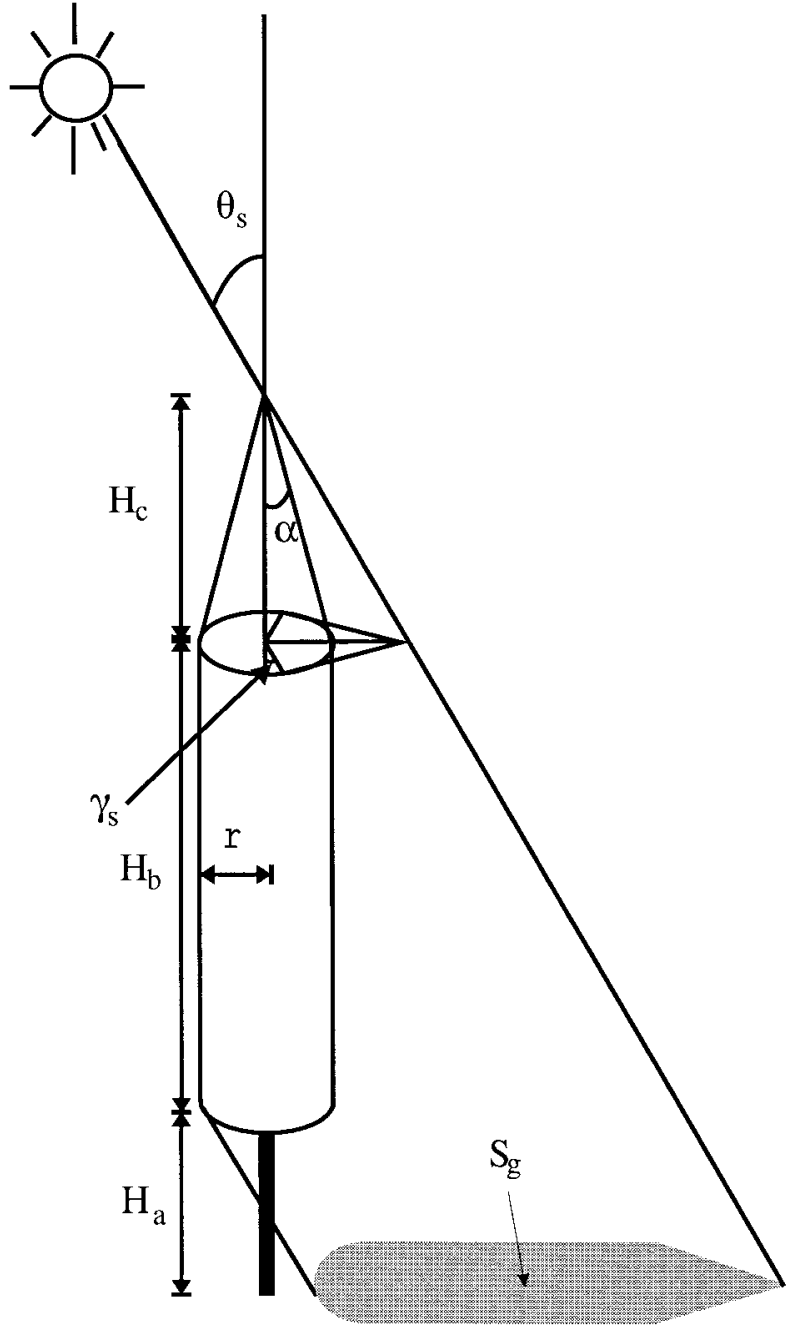

Fig. 5. Tree crown geometry and the definition of variables used.

[19]

$$
S_{g c}=\left[\frac{1}{\tan \left(\gamma_{s}\right)}-\frac{\pi}{2}+\gamma_{s}\right] r^{2}
$$

where $\gamma_{s}$ (see Fig. 5) is defined as

$$
\gamma_{s}=\sin ^{-1}\left(\frac{\tan (\alpha)}{\tan \left(\theta_{s}\right)}\right)
$$

where $\theta_{s}$ is the solar zenith angle. The shadow cast by the cylinder on the ground has an area given by

$$
2 \tan \left(\theta_{s}\right) H_{b} r
$$

To obtain the total area of a tree projected on the ground surface, the base of the tree, a disc, should also be included:

$$
\pi r^{2} .
$$

In this model, the cylinders can either rest on the ground surface or have their bases elevated to the same height $\left(H_{a}\right)$. Since the lower part of a forest is generally a trunk space without much foliage, it is more realistic to elevate the bases, i.e., to have the crown "on a stick." In our model, the height of tree crown base affects the contribution of the ground surface 
to the hotspot. The summation of (6), (8), and (9) gives the total area within the outline of a tree crown projected on the ground surface:

$$
S_{g}=\left\{\begin{aligned}
& \pi r^{2}+2 \tan \left(\theta_{s}\right) H_{b} r \text { for } \theta_{s} \leq \alpha \\
& {\left[\frac{1}{\tan \left(\gamma_{s}\right)}+\frac{\pi}{2}+\gamma_{s}\right.} \\
&+2 \tan \left(\theta_{s}\right) H_{b} r \text { for } \theta_{s}>\alpha
\end{aligned}\right.
$$

This projected area can be used to find the ground area not seen by a viewer, if the viewer is far enough so that the parallax won't change the view. For this reason, we only need to replace $\theta_{s}$ in (10) with $\theta_{v}$. This quantity is denoted by $V_{G}$, which is the area on the ground covered by opaque crowns in the viewer's direction:

$$
V_{g}=\left\{\begin{array}{cc}
\pi r^{2}+2 \tan \left(\theta_{v}\right) H_{b} r & \text { for } \theta_{v} \leq \alpha, \\
\frac{1}{\tan \left(\gamma_{v}\right)}+\frac{\pi}{2}+\gamma_{v} \\
+2 \tan \left(\theta_{v}\right) H_{b} r & \text { for } \theta_{v}>\alpha
\end{array}\right.
$$

After calculating the shadow area that a single tree casts on the ground, the sunlit crown proportion seen by the viewer is computed from the total surface area of the tree visible to the viewer projected to a plane perpendicular to the view line. For the cylinder we have

$$
t_{a b}=2 r \sin \left(\theta_{v}\right) H_{b}
$$

The total tree surface area projected on a plane perpendicular to the view line is

$$
t_{a c}= \begin{cases}\pi r^{2} & \text { for } \theta_{v}=0, \\ \pi r^{2} \cos \left(\theta_{v}\right) & \text { for } \theta_{v}<\alpha, \\ \pi r^{2} \cos \left(\theta_{v}\right)+t_{a c t} & \text { for } \theta_{v}>\alpha .\end{cases}
$$

$t_{\text {act }}$ of (13) is described in the Appendix.

For cylinders, the self-shadowing and illumination geometry are very simple: half the surface is illuminated and half in shadow. The illumination part seen by the viewer is then

$$
t_{i b}=2 r \sin \left(\theta_{v}\right) H_{b} \cdot\left[1-\frac{\phi}{\pi}\right]
$$

where $\phi$ is the azimuth angle difference between the sun and the viewer. The sunlit part of a cone seen by a viewer, denoted $t_{i c}$, cannot be easily expressed in a simple equation, but is described in the Appendix. This quantity depends on the apex angle of the trees and the positions of the sun and the viewer. If $\theta_{s}$ is smaller than $\alpha$, no self-shadow occurs on the cone, but as $\theta_{s}$ gets larger than $\alpha$, a portion of the cone is shaded. The proportion of the tree that is illuminated and seen by the viewer $\left(P_{t i}\right)$ can be found by adding the areas illuminated and dividing by the total surface area of the tree presented to the viewer. However, the contributions from both the cone and the cylinder part of a tree must be included. Thus

$$
P_{t i}=\frac{t_{i c}+t_{i b}}{t_{a c}+t_{a b}}
$$

\section{Canopy Gap Fraction}

The canopy gap fraction determines the contribution of the underlying surface to the reflectance measured above the canopy. It is based on the method described above for calculating the shadow area. If the trees are distributed randomly within the domain and the tree crowns are opaque, the probability of seeing the ground is

$$
P_{v g-r}=\left[1-\frac{V_{g}}{A}\right]^{D / n}
$$

where $D$ is the number of trees in the domain $B$ and $A=B / n$ is a quadrat. By definition there are $n$ quadrats in the domain. If the trees are clustered, the probability $P_{N}(i)$, found in (3), is used to compute this effect. We have

$$
P_{v g-c}=\sum_{i=0}^{\kappa} P_{N}(i)\left[1-\frac{V_{g}}{A}\right]^{i}
$$

where $i$ is the number of trees in $A$, and $P_{N}(i)$ is the probability of having $i$ trees in $A$. In this equation, gaps within tree crown are not considered. $P_{v g-c}$ represents the ground that can be seen between tree crowns. If we allow gaps in crowns and overlapping of crowns to occur, (17) becomes

$$
P_{v g}=\sum_{j=1}^{\kappa} P_{t j}\left(V_{g}\right) P_{\mathrm{gap}}^{j}\left(\theta_{v}\right)+P_{t 0}
$$

where

$$
P_{\text {gap }}^{j}\left(\theta_{v}\right)=\prod_{1}^{j} P_{\text {gap }}\left(\theta_{v}\right)
$$

is the gap probability (depending on $\theta_{v}$ ) inside the trees, and $P_{t j}\left(V_{g}\right)$, the probability of having $j$ trees intercepting the view line is calculated with a negative binomial:

$$
P_{t j}\left(V_{g}\right)=\sum_{i=j}^{\kappa} P_{N}(i)\left[\frac{(i+j-1) !}{(i-1) ! j !}\right]\left[1-\frac{V_{g}}{A}\right]^{i}\left[\frac{V_{g}}{A}\right]^{j}
$$

where $P_{N}(i)$ is the Neyman distribution (3) and $\kappa$ is an integer which should be large enough to consider all overlapping of the trees in a quadrat. It must correspond to $P_{N}(\kappa)=0$ or $P_{t(j=\kappa)}\left(V_{g}\right)=0$. In this paper $\kappa=350$. In the case of $j=0, P_{t 0}$ is equal to (17). Likewise the probability of the ground surface being illuminated, $P_{i g}$, can be found by replacing $V_{g}$ by $S_{g}$ and $\theta_{v}$ by $\theta_{s}$. In real forests, trees found in clusters are usually smaller than the average tree size. In one quadrat, we have $m=m_{1} m_{2}=D / n$ trees on average. The model assumes that for the probability of having more than $m$ trees in a quadrat, the size of the trees will decrease inversely with the number of trees per quadrat:

$$
V_{g i}=V_{g} \cdot m / i .
$$

With the setting used in the first section, $m=75$, the probability of having 100 trees in a quadrat, the individual tree shadow area is reduced by $75 \%$. For $i$ less than or equal to 75 all trees have the same size. 
The gap probability, $P_{\text {gap }}(\theta)$, is well known for a continuous medium [28]. For a discontinuous canopy, we use an equation similar to $\mathrm{Li}$ and Strahler [21] but modified to consider the foliage clumping effect [4]:

$$
P_{\text {gap }}\left(\theta_{s}\right)=e^{-G\left(\theta_{s}\right) L_{o} \cdot \Omega_{E} / \gamma_{E}}
$$

where

$$
L_{o}=\mu \cdot \bar{s}
$$

and

$$
\mu=L /[V \cdot D / B]
$$

is the foliage density, and

$$
\bar{s}\left(\theta_{s}\right)=\frac{V}{S_{g}\left(\theta_{s}\right) \cos \left(\theta_{s}\right)}
$$

is the mean path length of the solar beam through a tree. $V$ is the volume contained within the tree crown outline, $D / B$ is the number of trees per $\mathrm{m}^{2}, \Omega_{E}$ is a clumping index of the shoots within tree crowns [4], $L$ is the leaf area index (LAI) defined as half the total needle area per unit ground surface area [4].

Equation (22) gives the probability of the solar beam passing through a single tree. Using $\theta_{v}$ instead of $\theta_{s}$ gives the probability of seeing the background through a single tree. For a canopy with a random foliage angle distribution, $G(\theta)=0.5$. For canopies with branch architecture [3], $G(\theta)=a-b \theta$, where $a$ and $b$ are positive constants. Adding a gap probability to tree crowns gives less shadow on the ground and more ground area seen by the viewer.

\section{Mutual Shadowing EfFect}

At large $\theta_{v}$ values, a viewer sees mostly the upper part of the trees. Without considering the height-dependent mutual shadowing effect, the model will underestimate the proportion of sunlit canopy $P_{t i}$ in view because the illuminated top part is less likely in shadow. In this module of our model, we only separate a tree crown into two parts: cone and cylinder, and calculate the mutual shadow effects for these two parts in two steps. In step one, the mutual shadowing effects among the cones can be found by allowing the projected conical areas to overlap. The probability of cone overlapping is obtained by summing (20) from $j=2$ to $\kappa$, based on the shadow area of a cone cast on the ground in the sun's direction $\left(S_{g c}\right)$ and in the viewer's direction $\left(V_{g c}\right)$.

$$
P_{s c}=\sum_{j=2}^{k} P_{t j}\left(S_{g c}\right)\left[1-P_{\text {gap }}\left(\theta_{s}\right)^{j}\right]
$$

is the overlapping probability for $S_{g c}$ and

$$
P_{v c}=\sum_{j=2}^{k} P_{t j}\left(V_{g c}\right)\left[1-P_{\text {gap }}\left(\theta_{v}\right)^{j}\right]
$$

is the overlapping probability for $V_{g c}$. In (26) and (27), $1-P_{\text {gap }}\left(\theta_{v}\right)^{j}$ is multiplied to discount the gaps within the crowns. In step two, the mutual shadowing effects on the cylindrical part are calculated as the difference between the total overlapping and the cone overlapping. The total overlapping effects, denoted by $P_{s}$ and $P_{v}$, are calculated by replacing $S_{g c}$ and $V_{g c}$ with $S_{g}$ and $V_{g}$ in (26) and (27), where $S_{g}$ and $V_{g}$ are the ground shadow areas of a single tree crown in the sun's and viewer's directions, respectively. After weighting the overlapping probabilities of the crown and cylinder parts by their respective areas, the mutual shadowing effects for the cylindrical (base) part are then

$$
P_{s b}=\frac{P_{s} \cdot S_{g}-P_{s c} \cdot S_{g c}}{S_{g b}}
$$

and

$$
P_{v b}=\frac{P_{v} \cdot V_{g}-P_{v c} \cdot V_{g c}}{V_{g b}}
$$

where $S_{g b}$ and $V_{g b}$ are the projected areas on the ground in the sun and view directions, respectively. For the calculation of the probability of viewing the sunlit portion of tree crowns, the quantities needed are the proportions of the tree crowns that are not overlapped under the various scenarios, i.e.,

$$
\begin{aligned}
& Q_{s c}=1-P_{s c} \\
& Q_{v c}=1-P_{v c} \\
& Q_{s b}=1-P_{s b} \\
& Q_{v b}=1-P_{v b} .
\end{aligned}
$$

If the illumination and viewing directions are far apart, $Q_{s c}$ and $Q_{v s}$, as well as $Q_{s b}$ and $Q_{v b}$, are considered to be independent. The products of the pairs, i.e., $Q_{v c} \cdot Q_{s c}$ and $Q_{v b}$. $Q_{s b}$, are the probabilities of actually seeing the illuminated part of the cones and cylinders, respectively. We must multiply $t_{i b}$, the illuminated area of one tree, by $Q_{v b} \cdot Q_{s b}$ in (15), and $t_{i c}$ by $Q_{v c} \cdot Q_{s c}$, and $t_{a c}$ and $t_{a b}$ in the same equation are multiplied by $Q_{v c}$ and $Q_{v b}$, respectively, to obtain the actual areas of the conical and cylindrical parts of one tree seen by a viewer.

Near the principal plane on the backscatter side, the illumination and observation of a sunlit area can occur in the same gap in the canopy. In such a case, the processes of illumination and viewing are correlated. The angle range over which the correlation occurs depends on the tree size and the average tree spacing. A function $f(\phi)$, depending on the difference in the azimuth angle between the sun and the viewer, is used to consider this correlation effect. Using the mean distance between tree centres [13] calculated by

$$
E(d)=\frac{1}{2 \sqrt{D / B}}
$$

we can determine the azimuthal angle range within which overlapping of one tree with the other occurs in both the illumination and view directions

$$
\tan \left(\theta_{E}\right)=\frac{2 r}{E(d)-2 r} .
$$

A linear equation is used between $\phi=0$ and $\phi=\theta_{E}$

$$
f(\phi)=1-\frac{\phi}{\theta_{E}},
$$


where $f(\phi)$ is a kernel with non zero values only when $0<\phi<\theta_{E}$. The overall mutual shadowing effects, $Q_{c}$ and $Q_{b}$, for the conical and cylindrical parts, respectively, are then given by

$$
Q_{c}=Q_{v c} \cdot Q_{s c}+f(\phi) \cdot\left[Q_{s v c}-Q_{v c} \cdot Q_{s c}\right]
$$

and

$$
Q_{b}=Q_{v b} \cdot Q_{s b}+f(\phi) \cdot\left[Q_{s v b}-Q_{v b} \cdot Q_{s b}\right]
$$

where $Q_{s v c}$ is the minimum of $Q_{s c}$ and $Q_{v c}$, and $Q_{s v b}$ is the minimum of $Q_{s b}$ and $Q_{s b}$. With considerations of these mutual shadowing effects, the probability of seeing illuminated tree crowns becomes

$$
P_{t i}=\frac{Q_{c} \cdot t_{i c}+Q_{b} \cdot t_{i b}}{Q_{v c} \cdot t_{a c}+Q_{v b} \cdot t_{a b}} .
$$

Much of the complexity of the mutual shadow calculations arises from the fact that the conical and the cylindrical parts have different geometry and have to be separately modeled. Since conifer trees are better represented using the combination of these two parts than the single cone shape, we believe that the formulation presented above is an improvement over previous models based on cone geometry when used for conifer forests.

\section{CANOPY GAP SIZE AND Hotspot}

The hotspot is a phenomenon that occurs when the observation and the illumination directions coincide within the same canopy gap [16]. At the hotspot, the viewer sees either sunlit foliage or the sunlit background, resulting in large observed reflectance factors. In this case, the probability of seeing the ground, $P_{v g}$, is equal to the probability of having the ground illuminated, $P_{i g}$, and the probability of seeing tree crowns, $P_{s t}=1-P_{v g}$, is equal to $1-P_{i g}$. As the viewer moves away from the hotspot, the view line and the solar beam are less likely to fall in the same canopy gap and $P_{i g}$ and $P_{v g}$ gradually become independent of each other. The product of the pair $\left(P_{i g}\right.$ and $\left.P_{v g}\right)$ then determines the probability of seeing the sunlit ground. However, around the hotspot, such simple calculations are invalid because of the correlation of these two processes. The importance of the correlation in determining the hotspot has been investigated by many modellers including Kuusk [18], who devised a correlation function based on the leaf size. We found that leaf size is irrelevant for the correlation in conifer canopies. Nilson and Peterson [29] used a correlation based on a characteristic gap size. In the present paper, we introduce a canopy gap size distribution function for the calculation of the correlation. The function closely relates the hotspot to the canopy attributes.

The theory of gap size distribution in plant canopies was first derived by Miller and Norman [25] and further investigated by Chen and Black [5] and Chen and Cihlar [6]. The accumulated gap size distribution can be calculated from

$$
F_{a t}(\lambda)=\left[1+L_{t} \frac{\lambda}{W_{t}}\right] e^{-L_{t}\left[1+\left(\lambda / W_{t}\right)\right]}
$$

for gaps $\geq \lambda$, where $W_{t}$ is the characteristic width of a tree crown projected on the ground, and $L_{t}$ is the clumpingadjusted projected tree crown area index. This formulation is made according to the finding of Chen and Cihlar [7] that a large part of the gap size distribution curve measured in boreal forests is determined by tree crowns as discrete objects. $W_{t}$ and $L_{t}$ are computed as

$$
W_{t}=\sqrt{S_{g}}
$$

and

$$
L_{t}=\Omega_{t} S_{g} D / B
$$

where $S_{g}$ is the area of a tree crown projected on the ground in the sun's direction and $\Omega_{t}$ is a tree clumping index, determined by the Neyman distribution as

$$
\Omega_{t}=\log \left(P_{i g}(\text { Neyman })\right) / \log \left(P_{i g}(\text { Poisson })\right)
$$

It is 0.95 obtained from simulation with a Neyman grouping of 4 for a forest with 4000 stems per hectare divided into 25 quadrats.

Similar to (40), a distribution for gaps inside crowns can be computed where the shoots are taken as the foliage elements

$$
F_{a s}(\lambda)=\left[1+L_{s} \frac{\lambda}{W_{s}}\right] e^{-L_{s}\left[1+\left(\lambda / W_{s}\right)\right]} .
$$

where

$$
L_{s}=\frac{G\left(\theta_{s}\right) \Omega_{E} L}{\gamma_{E} \cos \left(\theta_{s}\right)}
$$

is the clumping-adjusted foliage area index and the characteristic width of the foliage elements is

$$
W_{s}=\sqrt{A_{s}} .
$$

$\Omega_{E}$ is a clumping index for the effect of confining shoots within tree crowns; $\gamma_{E}$ is the needle-to-shoot area ratio quantifying the effect of needle grouping in shoots on the radiation interception; and $A_{s}$ is the average shoot projected area. According to Chen [2], for the black spruce stand investigated in this paper, $L=4.5, G(\theta)=0.5, \Omega_{E}=0.70, \gamma_{E}=1.41$ and $W_{s}=35 \mathrm{~mm}$. Only very small gaps are computed inside trees, but they contribute significantly to the total canopy gap fraction because of their large numbers. In the calculation of the hotspot, these small gaps are of critical importance in determining the shape of the hotspot.

Fig. 6 shows the accumulated gap size distribution measured along a $300 \mathrm{~m}$ transect in the black spruce forest [2]. The measured gap size distribution can be separated into two parts: between and within the tree crowns. Equations (40) and (44) are used to simulate these two parts respectively. The parameters used in (40) and (44) are those found in Table I except $W_{t}$ that was fixed at $1.4 \mathrm{~m}$ to reflect the typical width of tree crowns encountered on the transect at about $45^{\circ}$ to the sun. The characteristic width $W_{s}$ for black spruce shoots was measured to be $3.5 \mathrm{~cm}$. Same $W_{t}$ and $W_{s}$ values were used for both gap size distributions, i.e., Fig. 6(a) and (b). In both cases, the contributions of gaps between and within tree crowns to the measured gap size distributions are distinct, but there is 


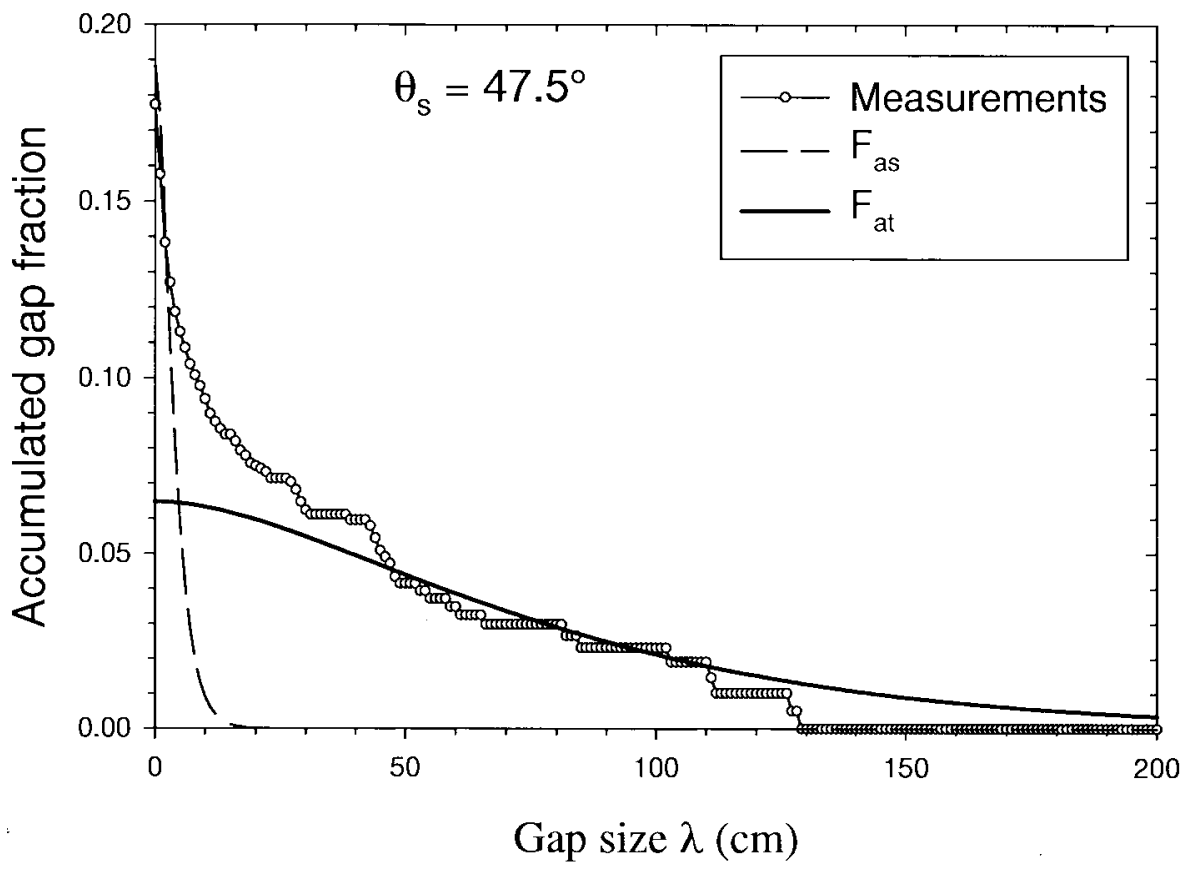

(a)

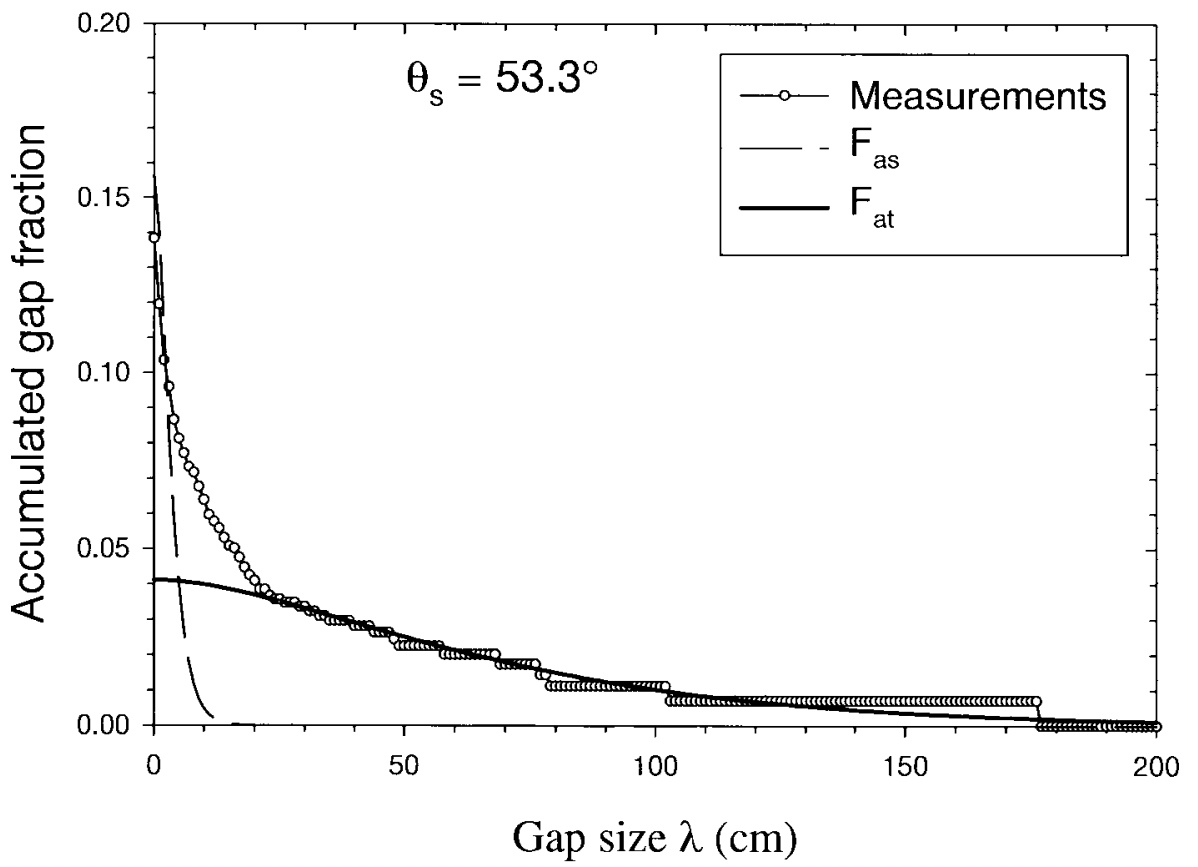

(b)

Fig. 6. Two gap size accumulation curves measured in the old black spruce stand using an optical instrument at two solar zenith angles. In both cases (a) and (b), the accumulation of the gap fraction resulting from gaps of various sizes can be separated into two components: gaps between $\left(F_{a t}\right)$ and within $\left(F_{a s}\right)$ tree crowns. The portion of the curve in the $\lambda$ range of $10-50 \mathrm{~cm}$ is due to branch architecture.

a portion of the distribution curve in the range of $10-50 \mathrm{~cm}$ which can not be fitted using the crown and shoot attributes. This out-lying portion is a result of the branch architecture because the gaps between branches are larger than those between shoots but smaller than those between tree crowns.

\section{A. Hotspot Between Crowns}

$F_{a t}(\lambda)$ and $F_{a s}(\lambda)$ are quantities that can be measured directly in a forest canopy. From the measurements of $F_{a t}(\lambda)$, the following gap size distribution can be derived:

$$
P_{t}(\lambda)=e^{-L_{t}\left[1+\left(\lambda / W_{t}\right)\right]} .
$$

$P_{t}(\lambda)$ is defined as the probability of a horizontal probe of length $\lambda$ falling completely within a gap between tree crowns. This gap size distribution between tree crowns is important in determining the contribution of the ground surface to the hotspot. Fig. 7(a) shows how $P_{t}(\lambda)$ varies with $\lambda$ at different solar zenith angles. For the calculation of the hotspot kernel 


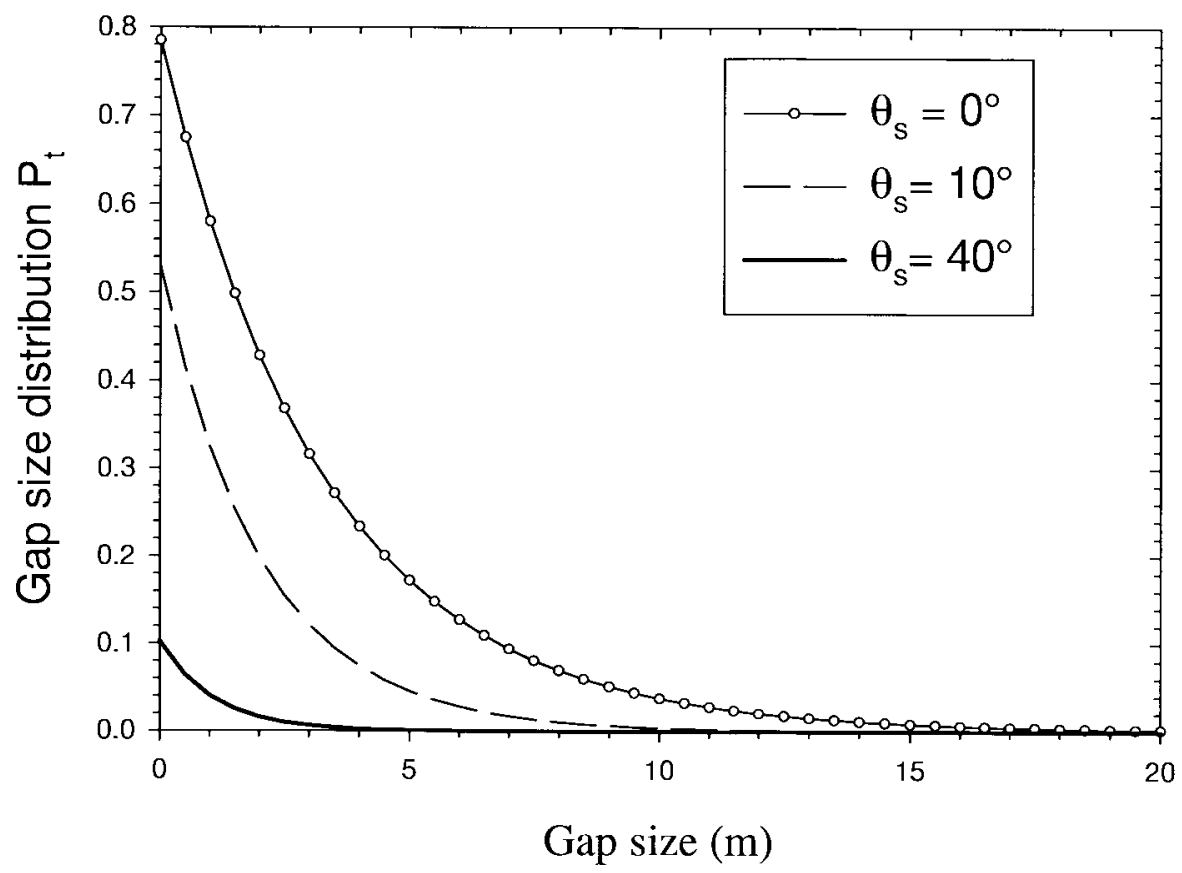

(a)

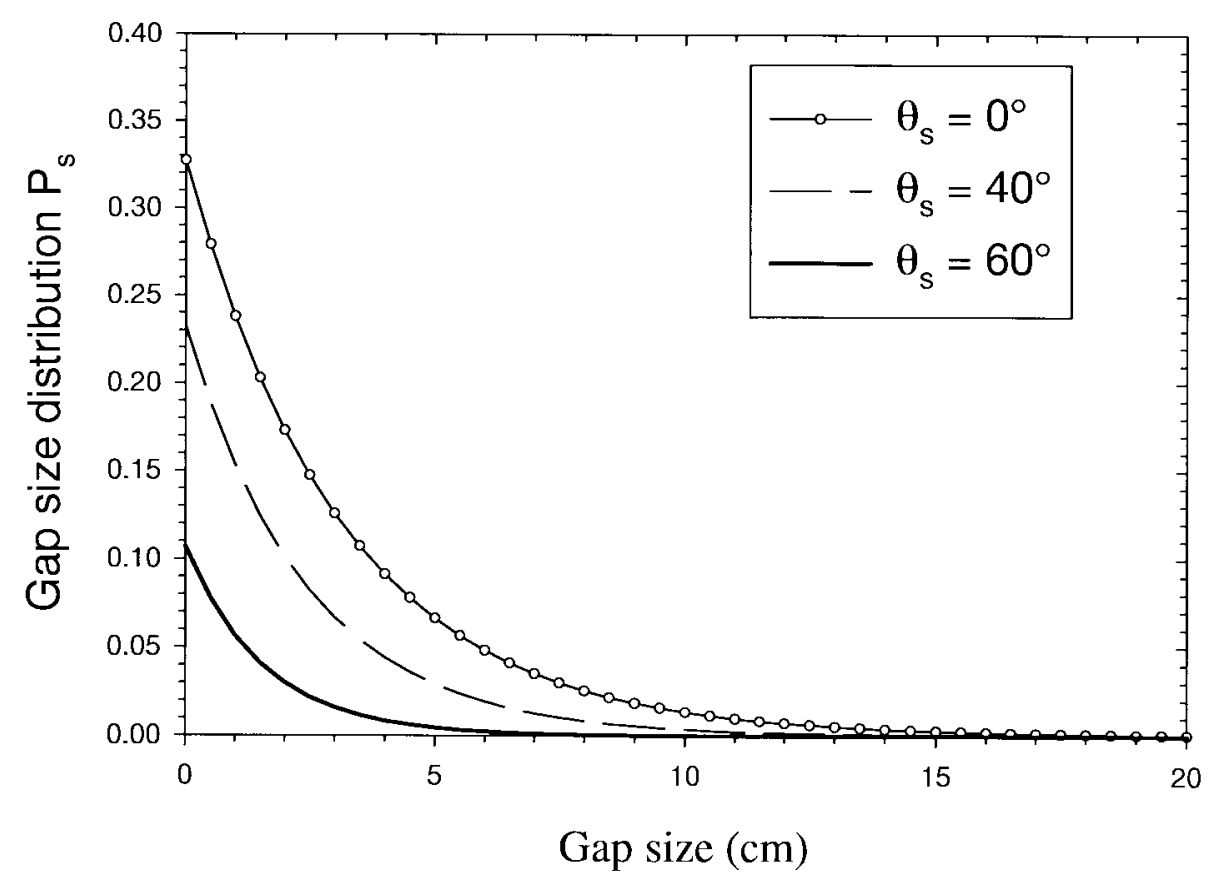

(b)

Fig. 7. Calculated gap size distributions in the black spruce stand at three solar zenith angles: (a) between tree crowns and (b) inside tree crowns. For the distributions between tree crowns, (47) is used with $L_{t}$ and $W_{t}$ from (41) and (42), respectively. For the distributions inside tree crowns, $L_{t}$ and $W_{t}$ are replaced by $L_{s}$ and $W_{s}$, respectively, where $L_{s}$ is calculated from (45) and $W_{s}$ is taken to $35 \mathrm{~mm}$.

for the ground, a gap number density function is used, which is defines as

$$
N_{t}(\lambda)=-\frac{d P_{t}(\lambda)}{d \lambda}=\frac{L_{t}}{W_{t}} e^{-L_{t}\left[1+\left(\lambda / W_{t}\right)\right]}
$$

The probability $P_{G}$ of observing the sunlit ground under the tree crowns can then be written in the general form:

$$
P_{G}=P_{i g} P_{v g}+\left[P_{i g}-P_{i g} P_{v g}\right] F_{t}(\xi),
$$

where $F_{t}(\xi)$ is a hotspot kernel, which is unity at the hotspot and zero when the illumination and view angles are far apart, and $\xi$ is the angle between the sun and the viewer determined by $\cos (\xi)=\cos \left(\theta_{s}\right) \cos \left(\theta_{v}\right)+\sin \left(\theta_{s}\right) \sin \left(\theta_{v}\right) \cos (\phi)$. The first term on the right hand side of (49) is the probability of observing the sunlit ground when $P_{i g}$ and $P_{v g}$ are not correlated, i.e., the viewer sees the sunlit ground through a gap different from that of illumination. The second term gives the additional probability resulting from the correlation. Fig. 8 
TABLE I

Model InPuTS for a Black Spruce CANopy

\begin{tabular}{c|c}
\hline$I_{a}$ & $0.5 \mathrm{~m}$ \\
\hline$\tilde{H}_{b}$ & $6.5 \mathrm{~m}$ \\
\hline Radius of crown & $0.45 \mathrm{~m}$ \\
\hline$G(\theta)$ & 0.5 \\
\hline LAI & 4.5 \\
\hline Dornain & 1 ha \\
\hline Density & 4000 tress $/$ ha \\
\hline Quadrat size & $400 \mathrm{~m}^{2}$ \\
\hline Neyman grouping & 4 \\
\hline$\alpha$ & $13^{\circ}$ \\
\hline$\gamma_{E}$ & 1.41 \\
\hline$\Omega_{E}$ & 0.70 \\
\hline$W_{s}$ & 0.035 \\
\hline$R_{G}$ (red) & 0.06 \\
\hline$R_{T}$ (red) & 0.13 \\
\hline$R_{Z G}$ (red) & 0.006 \\
\hline$R_{Z T}$ (red) & 0.01 \\
\hline$R_{G}$ (nir) & 0.20 \\
\hline$R_{T}$ (nir) & 0.53 \\
\hline$R_{Z G}$ (nir) & 0.05 \\
\hline$R_{Z T}$ (nir) & 0.08 \\
\hline
\end{tabular}

shows how a gap of certain size contributes to the hotspot kernel. The angle range in which the viewer can see the sunlit ground through the same gap as the illumination is determined by the gap size and the effective height of the gap column that depends on the view zenith angle. For one gap of size $\lambda$, we have the hotspot function

$$
F_{t}(\xi)=1-\frac{\xi}{\tan ^{-1}(\lambda / H)}
$$

where $H=\left[H_{a}+H_{b}+H_{c} / 3\right] / \cos \left(\theta_{s}\right)$. For the whole canopy containing multiple gaps of different sizes with a number density of $N(\lambda)$, the hotspot function is obtained through the following integration:

$$
F_{t}(\xi)=\frac{\int_{\lambda_{\min }}^{\infty}\left[1-\frac{\xi}{\tan ^{-1}(\lambda / H)}\right] N(\lambda) d \lambda}{\int_{\lambda_{\min }}^{\infty} N(\lambda) d \lambda} .
$$

For a given angle difference between the sun and the viewer, there is a minimum gap size $\lambda_{\min }$ in which the view line penetrates through the same gap as the solar beam. It is determined by

$$
\lambda_{\min }=H \tan (\xi) .
$$

The ground reflectance is assumed to be isotropic in this paper.

\section{B. Hotspot within Tree Crowns}

In the estimation of the hotspot within the tree crown, a gap size distribution within individual tree crowns is used. In previous geometric-optical models, the imaginary tree crown surface has been treated as a smooth surface in the calculation of the hotspot [22]. Some more elaborate models have dealt with the crowns as object containing turbid media with multiscattering [29], [23]. Since shadows can be observed on the sunlit side of real tree crowns, the microscale structures within tree crowns have important contributions to the hotspot,

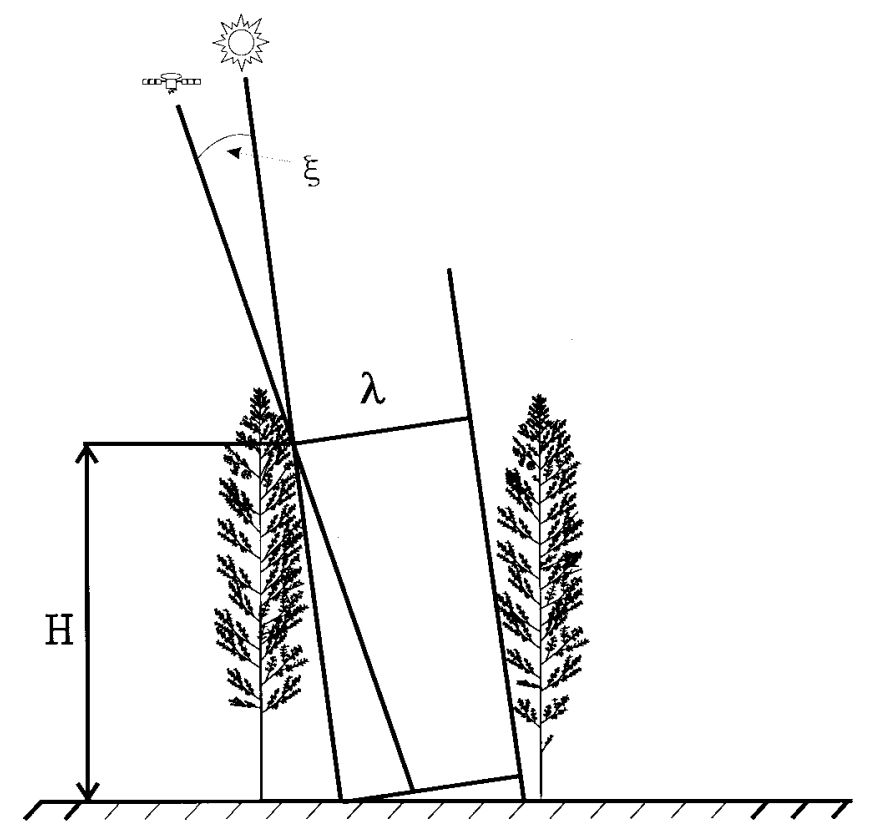

Fig. 8. Geometry determining the hotspot on the ground due to the co-occurrance of illumination and observation within the same gap between tree crowns.

modifying the hotspot shape and magnitude. An important structure within conifer trees is the shoot, which is the basic collection of needles. Needles are grouped tightly in shoots, which allow little radiation penetration and can be treated as the basic foliage units for radiation modeling [7]. Similar to the distribution between crowns, a gap size distribution within the crown is used to calculate the gap number density function

$$
N_{s}(\lambda)=-\frac{d P_{s}(\lambda)}{d \lambda}=\frac{L_{s}}{W_{s}} e^{-L_{s}\left[1+\left(\lambda / W_{s}\right)\right]} .
$$

The formulation presented above has implications on the inversion of the model for leaf area index because the microscale structures within the tree crowns affect the BRD and cannot be ignored.

In computing the hotspot within the tree crowns, the selfshadowing and the vertical tree crown structure must be considered. Discontinuities in mathematical expressions are found at nadir or the boundary between sunlit and shaded sides of a crown. Therefore, we treat the two sides separately. On the sunlit side, the probability of seeing the illuminated foliage $P_{T}$ at the hotspot is simply $1-P_{i g}$, i.e., the viewer sees either the sunlit ground or the sunlit foliage. Considerable complication arises when we treat the tree crown surface as a complex surface with microscale structures. In this case, a view line can penetrate into the depth of the crown and reach to the shaded foliage behind the sunlit part even though the imaginary tree crown surface is theoretically sunlit. The problem may be understood in the extreme cases: 1) a tree crown is very dense-the imaginary surface can reasonably represent the tree crown, and 2) a tree crown is very sparse - the imaginary surface ceases to have meaning. The reality is in between these two extremes, i.e., the imaginary surface exists but is unsmooth or complex. In this case, the effect of mutual shadowing among foliage elements within a tree crown cannot be ignored. 


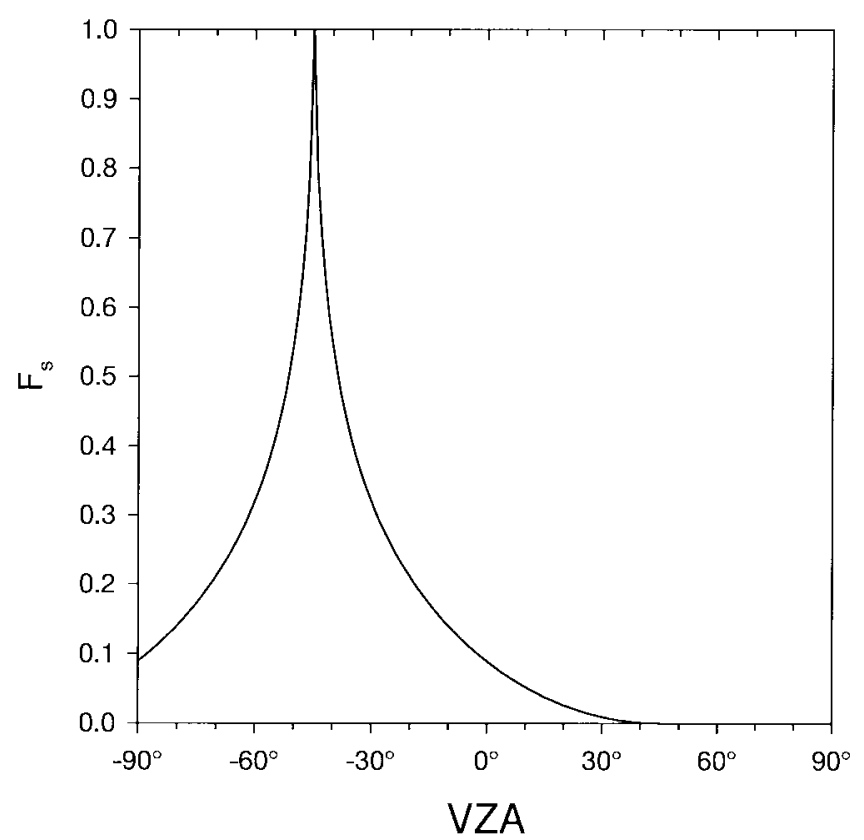

(a)

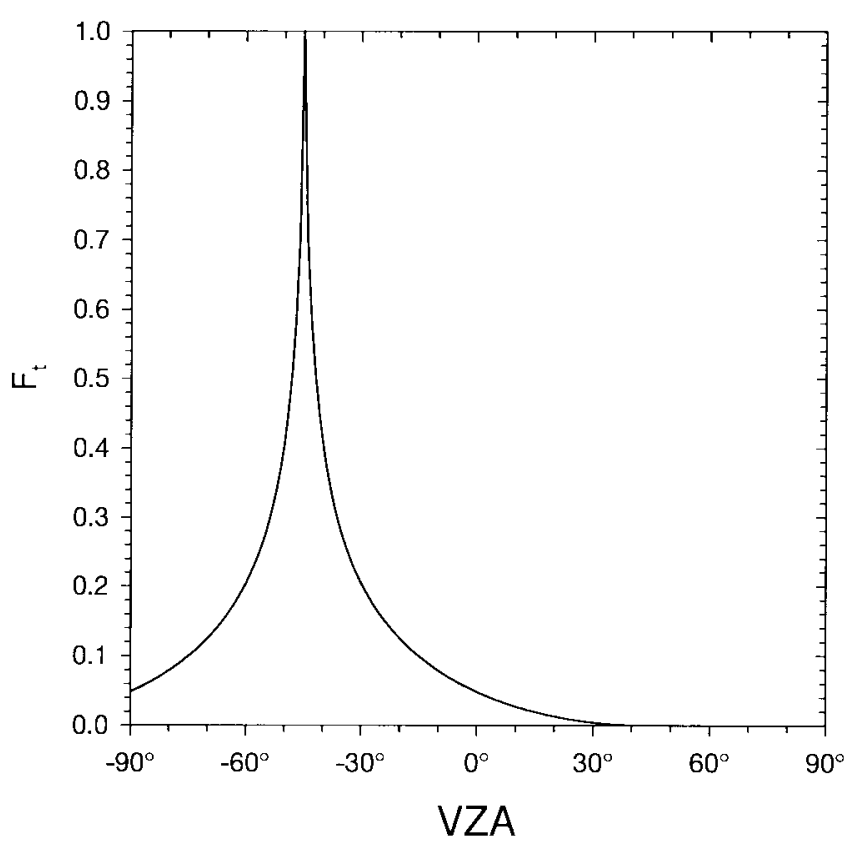

(b)

Fig. 9. Examples of (a) the ground and (b) the tree crown hotspot kernel for $\theta_{s}=45^{\circ}$ calculated using (51) and (67) with parameters given in Table I.

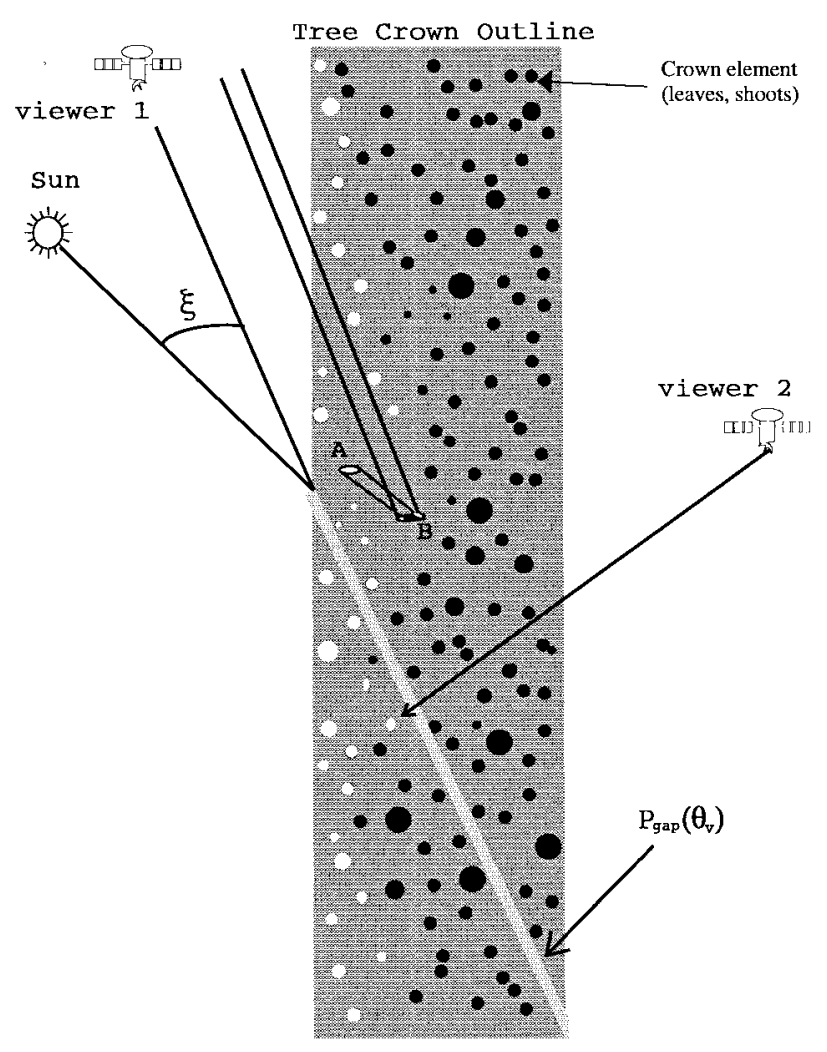

Fig. 10. Gap probability used in modeling the probability of observing sunlit foliage inside a tree crown from the sunlit side $\left(Q_{1}\right.$ for viewer 1$)$ and from the standard side $\left(Q_{2}\right.$ for viewer 2$)$.

Fig. 10 shows the physics involved in the determination of the hotspot within a tree crown, where a tree is considered as a vertical structure with spherical foliage elements (shoots) dispersed within it. Using $L(x)$ as the accumulated LAI from the imaginary surface to a given location $x$ within the crown, the accumulated sunlit leaf area from 0 to $L(x)$ is $1-e^{-C_{s} L(x)}$, where $C_{s}=G\left(\theta_{s}\right) \Omega_{E} / \gamma_{E} \sin \left(\theta_{s}+\alpha\right)$ to take into account the cone inclination and the vertical structure. At position $x$, the increment of sunlit leaf area with increasing $L(x)$ is

$$
\frac{d}{d L(x)}\left[1-e^{-C_{s} L(x)}\right]=C_{s} e^{-C_{s} L(x)} .
$$

Similarly, the increment of leaf area seen after $d L(x)$ is

$$
\frac{d}{d L(x)}\left[1-e^{-C_{v} L(x)}\right]=C_{v} e^{-C_{v} L(x)} .
$$

If we denote $L_{H}$ as the leaf area index accumulated horizontally from the sunlit side to the shaded side, the total probability of viewing sunlit leaf area from 0 to $L_{H}$ is then

$$
\begin{aligned}
Q_{1}\left(L_{H}\right) & =\Gamma(\xi) \int_{0}^{L_{H}} C_{v} C_{s} e^{-C_{v} L(x)} e^{-C_{s} L(x)} d L(x) \\
& =\Gamma(\xi)\left[1-e^{-L_{H}\left(C_{s}+C_{v}\right)}\right]\left[\frac{C_{s} C_{v}}{C_{s}+C_{v}}\right]
\end{aligned}
$$

where $\Gamma(\xi)$ is the first-order scattering (geometric shadow) phase function of the foliage elements. It is defined as

$$
\Gamma(\xi)=\left[1-\frac{C_{p} \xi}{\pi}\right]
$$

where $C_{p}$ is a coefficient determined by the optical properties of foliage elements. If the elements are solid spheres with a Lambertian surface, $C_{p}$ is unity, resulting in the phase function being 1.0 at $\xi=0,0.5$ at $\xi=\pi / 2$ and 0 at $\xi=\pi$. The phase function in this case gives the proportion of the sunlit sphere surface seen by the viewer. Although a conifer shoot can be approximated by a sphere to describe the projected area [2], it can not be treated as a solid because of the gaps within it. The value of $C_{p}$ for porous elements is smaller than unity. Because of lack of data, we assume that $C_{p}=0.75$ in this paper. This 
constant is found to affect the sharpness of the hotspot. $Q_{1}$ must be calculated for all trees, it gives

$$
Q_{1 \mathrm{tot}}=\sum_{j=1}^{\kappa} Q_{1}\left(j \cdot L_{H}\right) \cdot P_{t j}\left(V_{g}\right)
$$

where $P_{t j}\left(V_{g}\right)$ is the probability of a view line going through $j$ tree crowns calculated by (20). On the shaded side, the probability of observing sunlit leaves also exists, especially in canopies with sparse tree crowns. The increment of sunlit leaf area along the sun's direction is calculated using (54), but the increment of leaf area viewed from the opposite side is calculated differently as follows:

$$
\frac{d}{d L(x)}\left[1-e^{-C_{v}\left(L_{H}-L(x)\right)}\right]=-C_{v} e^{-C_{v}\left(L_{H}-L(x)\right)} .
$$

At a given depth $x$, the probability of observing sunlit foliage is

$$
\Gamma(\xi) C_{s} e^{-C_{s} L(x)}\left[-C_{v} e^{-C_{v}\left(L_{H}-L(x)\right)}\right] .
$$

After integration from $L_{H}$ to 0 across the tree crown, the overall probability becomes

$$
Q_{2}\left(L_{H}\right)=\left[1-\frac{C_{p} \xi}{\pi}\right]\left[e^{-C_{s} L_{H}}-e^{-C_{v} L_{H}}\right]\left[\frac{C_{s} C_{v}}{C_{v}-C_{s}}\right] .
$$

This phenomenon must be considered on each tree along the path of view. On the first tree, it is simply $Q_{2}\left(L_{H}\right)$. For $i$ trees along the path it is

$$
Q_{2 \mathrm{tot}}=Q_{2}\left(L_{H}\right) \sum_{i=1}^{\kappa} \cdot P_{t t}(i) \cdot K(i)
$$

where

$$
P_{t t}(i)=P_{a t}(i) P_{a t}(i-1) P_{\text {gap }}^{i-1}\left(\theta_{v}\right)
$$

in which

$$
P_{a t}(i)=\sum_{j=i}^{\kappa} P_{t j}\left(V_{g}\right) .
$$

$P_{a t}(i)$ represents the probability of having $i$ trees within one path. The probability of seeing the $i$ th tree behind $i-1$ trees is found by the probability of having $i-1$ tree overlapping, $P_{a t}(i-1)$, times the probability of passing through $i-1$ trees, $P_{\text {gap }}^{i-1}\left(\theta_{v}\right)$. As the view line penetrates through the forest, it reaches the lower portion of the canopy where shaded foliage is more likely to be observed. For the $i$ th tree on the path, this height attenuation is considered by

$$
K(i)=e^{-i G\left(\theta_{v}\right) \cdot S L_{E} \cdot \cos \left(\theta_{v}\right) / \cos \left(\theta_{s}\right) \delta_{E}} .
$$

With the proportion of sunlit and shaded tree crown as $P_{t i}$ and $\left(1-P_{t i}\right)$ respectively, we can then calculate the probability of seeing the illuminated foliage as

$$
P_{T f}=P_{t i} \cdot Q_{1 \mathrm{tot}}+\left[1-P_{t i}\right] \cdot Q_{2 \mathrm{tot}} .
$$

This equation is only valid far from the hotspot. A hotspot function, similar to (51), can also be defined. $F_{s}(\xi)$ follows the same principles as $F_{t}(\xi)$ but it involves foliage elements (shoots) instead of tree crowns.

$$
F_{s}(\xi)=\frac{\int_{\lambda_{\min _{s}}}^{\infty}\left[1-\frac{\xi}{\tan ^{-1}\left(\lambda / H_{s}\right)}\right] N_{s}(\lambda) d l}{\int_{\lambda_{\min _{s}}}^{\infty} N_{s}(\lambda) d \lambda}
$$

where $H_{s}$ is the effective distance between two layers of leaves inside a tree crown. In our model, it is inversely proportional to the leaf area density in the tree crown or $L_{o}$, i.e.,

$$
H_{s}=\frac{r}{L_{o}}
$$

where $r$ is the tree crown radius. This definition produces sharper hotspots in canopies with sparser tree crowns. After considering the hotspot on tree crowns, the overall probability of observing sunlit foliage is

$$
P_{T}=P_{T f}+\left[\left(1-P_{i g}\right)-P_{T f}\right] F_{s}(\xi) .
$$

This gives $P_{T f}$ outside of the hotspot where $F_{s}(\xi)=0$ and $\left(1-P_{i g}\right)$ at the centre of the hotspot where $F_{s}(\xi)=1$. Fig. 9 shows examples of the hotspot kernel calculated using (51) and (67) along the principal solar plane at a solar zenith angle of $45^{\circ}$. As the first approximation, the shape of the kernel is the same in all directions, i.e., the hotspot has a circular shape.

\section{Canopy Reflectance}

For estimating the first order scattering, the model computes the various components: sunlit foliage $\left(P_{T}\right)$, sunlit ground $\left(P_{G}\right)$, shaded foliage $\left(1-P_{v g}-P_{T}\right)$ and the shaded ground $\left(P_{v g}-P_{G}\right)$. If direct solar beams are the sole source of illumination and no multiple scattering occurs, only the first two components are responsible for the reflectance of the canopy. However, since the diffuse radiation from the sky is considerable compared with the direct radiation and the multiple scattering is also inevitable, the shaded components cannot be ignored. These components are considered in our model by assigning the appropriate reflectivities to them as follows:

$$
R_{Z T}=C_{m} \cdot F_{d t} \cdot R_{T}
$$

and

$$
R_{Z G}=C_{m} \cdot F_{d g} \cdot R_{G},
$$

where $R_{Z T}$ and $R_{Z G}$ are the reflectivities for shaded foliage and ground, respectively; $R_{T}$ and $R_{Z}$ are the reflectivities for sunlit foliage and ground, respectively; $F_{d t}$ and $F_{d g}$ are the fractions of diffuse irradiance in the total incoming solar irradiance above and below the stand, respectively; and $C_{m}$ is a multiple-scattering factor. $R_{Z T}$ and $R_{Z G}$ can also be measured directly from a forest stand. The total canopy reflectance is

$$
R=R_{T} \cdot P_{T}+R_{G} \cdot P_{G}+R_{Z T} \cdot Z_{T}+R_{Z G} \cdot Z_{G}
$$

where $Z_{T}=1-P_{v g}-P_{T}$ and $Z_{G}=P_{v g}-P_{G}$. All these reflectivities are wavelength dependent. In modeling canopy reflectance in red and near-infrared bands, (72) is used with a different value for each reflectivity. 


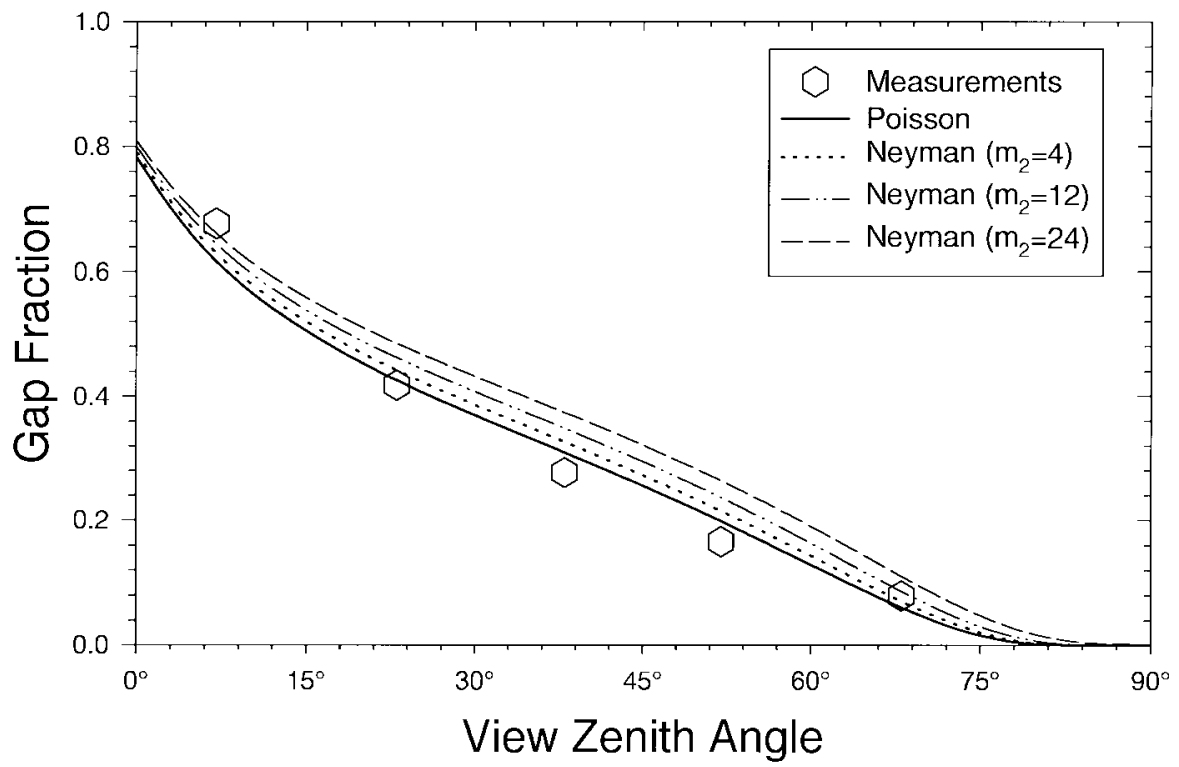

Fig. 11. Canopy gap fraction modeled with different Neyman group sizes as compared with the measurements from a black spruce forest.

\section{Model Results}

\section{A. Effect of Neyman Grouping}

The model first computes the canopy gap fraction $P_{v g}(18)$. Measurements from a boreal black spruce forest were used to validate the computation. According to Chen [2], the following values are used: 4000 for the tree density (stems per hectare), $6.5 \mathrm{~m}$ for the average tree height, 4.5 for LAI, and $0.45 \mathrm{~m}$ for the crown radius. The quadrat size is fixed at $400 \mathrm{~m}^{2}$, having an average of 200 trees per quadrat. Fig. 11 shows $P_{v g}$ distributions calculated using the Poisson model and the Neyman model with groupings of four, 12 , and 24 . Near the vertical view direction, the different sizes of Neyman grouping do not have much effect because the forest is very open. The effect of the grouping is more pronounced for $\theta_{v}$ between $15^{\circ}$ and $60^{\circ}$. The best fits are found with small Neyman groups. An analysis of 24 quadrats of $10 \times 10 \mathrm{~m}^{2}$ each measured in black spruce stands gives $m_{2}=2$ calculated with (5), but when grouped into six $400 \mathrm{~m}^{2}$ quadrats, $m_{2}=4$. A grouping of 4 is used in all simulations unless noted otherwise.

The measured gap fraction data at large zenith angles are positively biased because of the effect of multiple scattering on the measurements using an optical instrument [2]. The modeled curves all show a sharp increase near the vertical direction because of the simple geometry to represent the tree crown and overlapping of the crowns without considering the repulsion effect. This creates unsmoothness of the model results at the nadir as shown later. In reality, the tree crown geometry is more variable and the reflectance distribution are usually smoother around nadir.

To understand the effect of the Neyman grouping, Fig. 12 shows different components of the model for a range of $m_{2}$ values from 1 to 50 at $\theta_{v}=30^{\circ}, \theta_{s}=55^{\circ}$ and $\phi=0$. The grouping of trees produces a larger gap fraction because it increases the probability of having quadrats with few trees. With the increasing grouping size, the proportion of canopy illuminated $\left(P_{t i}\right)$ decreases, and so does the probability of seeing a tree illuminated $\left(P_{T}\right)$. This is mainly because of the increase in the gap fraction. In accordance with $P_{v g}$, the probability of seeing the illuminated ground surface $\left(P_{G}\right)$ increases. Red and near infra-red reflectances decrease with the Neyman grouping because ground reflectivity is low compared to the crown reflectivity.

\section{B. BRD and Hotspot}

The plots in Fig. 13 were computed using the same inputs as in Fig. 12 and the reflectivities summarized in Table I. Based on measured spectra (White et al. [36], Middleton et al. [24], Soffer [32]), $R_{G}=0.06$ and $R_{T}=0.13$ for the red band and $R_{G}=0.20$ and $R_{T}=0.53$ for the nearinfrared band. The ground reflectivity in the red band is smaller than that of leaves even though the overall reflectivity of the stand is lower than the ground reflectivity because of the shadow components in the stand. Using (70) and (71) we can attribute the appropriate reflectivities to the shaded tree crown and ground surfaces. Because of the small contributions from shaded ground and crowns, we use constant multiscatterring factors, leading to $C_{m} \cdot F_{d t}=0.08$ and $C_{m} \cdot F_{d g}=0.10$ for the red band and $C_{m} \cdot F_{d t}=0.15$ and $C_{m} \cdot F_{d g}=0.25$ for the near infra-red band. The six plots in Fig. 13 show the same components as those in Fig. 12 but as distributions against $\theta_{v}$ on the principal solar plane at $\theta_{s}=35^{\circ}$. For the view angle $\theta_{v}$, we use the following sign convention: negative for backscatter and positive for forwardscatter. Fig. 13(a) shows the gap fraction versus the view zenith angle for a Neyman grouping of 4. Fig. 13(b) is the proportion of the imaginary tree crown surface seen by the viewer that is illuminated. From $\theta_{v}=-90^{\circ}$ to $-35^{\circ}$ it equals unity, meaning that all tree crowns imaginary surface in view are illuminated in the principal solar plane. At nadir $\left(\theta_{v}=0\right)$, there is a discontinuity because the viewer can only see the cone part of the tree crown which is mostly illuminated. On the backscattering 

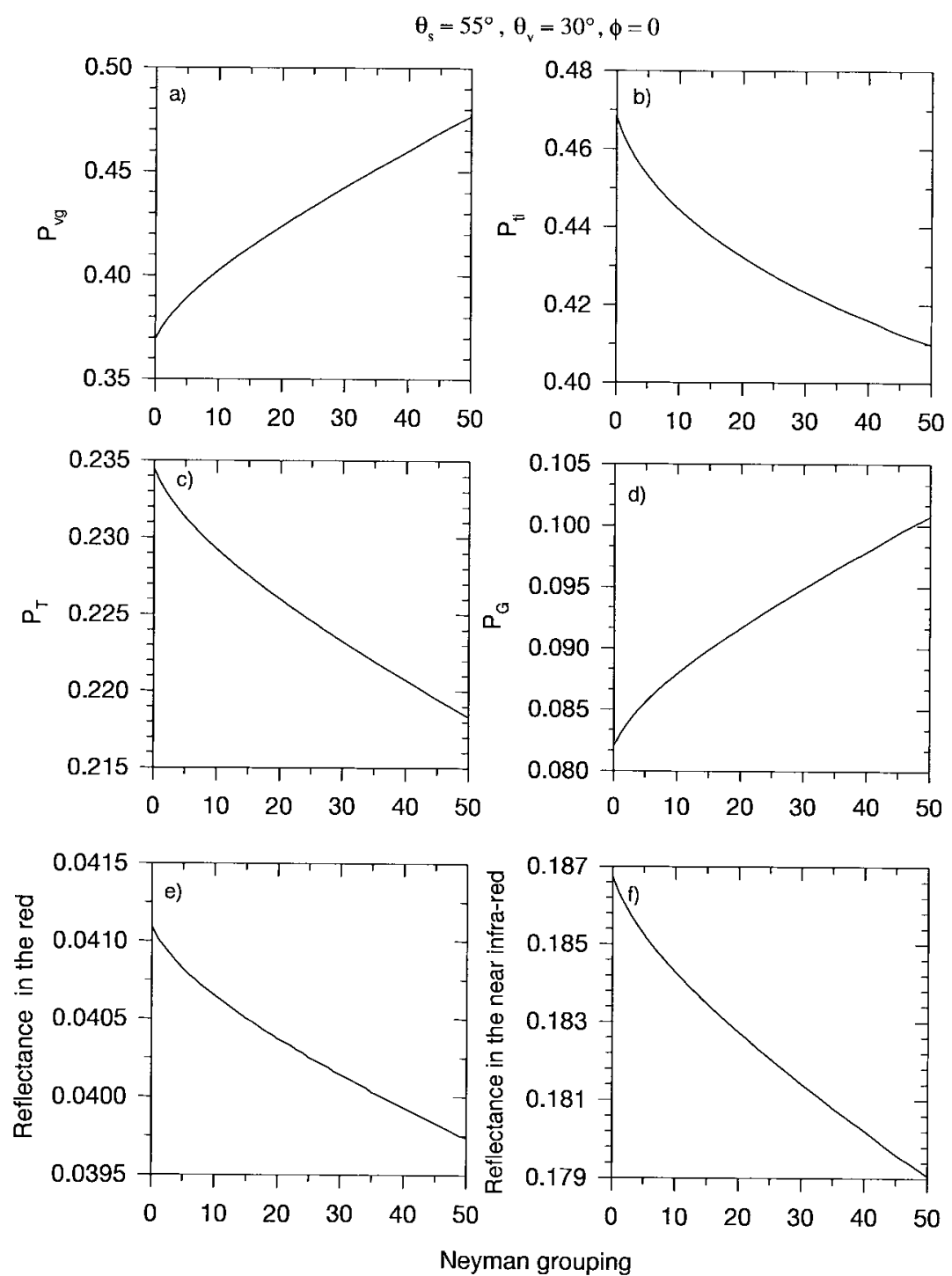

Fig. 12. Different components of the model versus the Neyman grouping at a view zenith angle of $35^{\circ}$ in the principle solar plane at a solar zenith angle of $55^{\circ}$.

side, $P_{t i}$ decreases near the vertical direction because the viewer can see the lower part of the cylinder that is shaded by other cylinders. On the fowardscattering side, the cylinder is completely shaded, and as $\theta_{v}$ increases, more of the shaded cylinder occupies the view. At very large $\theta_{v}$ values, the conical part dominates the view, and the proportion of the tree in view that is illuminated increases because more than half of the cone surface is usually sunlit. Fig. 13(c) represents the probability of seeing illuminated foliage within tree crowns. It includes the probability of observing sunlit foliage from the shaded side and the hotspot effect on the illuminated side. A pronounced hotspot peak is computed at $\theta_{v}=35^{\circ}$. Fig. 13(d) is the probability of seeing the ground illuminated by the sun. The bi-module distribution pattern results from the peaks at the hotspot and at nadir. The peak at the nadir is due to the largest gap probability at that angle. The other peak at the hotspot is obtained after the introduction of the hotspot function (51), otherwise the $P_{G}$ curve would be symmetric about the centre. At the hotspot, all the ground areas in view are illuminated, and the value of $P_{G}$ is simply $P_{v g}$ at the same angle $(0.35$ at $\theta_{v}=35^{\circ}$ ). Figs. 13(e) and 13(f) describe the BRD in the red and near-infrared bands. Both distributions resemble the probability of seeing illuminated foliage $\left(P_{T}\right)$ because of the large $P_{T}$ values and the large reflectivity values for the foliage. Although the magnitudes of the red and near-infrared reflectances are very different, the shapes of the distributions are remarkably similar because only the first-order scattering is considered in the calculation and the multiple scattering effects are included as invariant offsets. However, there are subtle differences in the shapes due to the different foliage and ground reflectivity combinations with $P_{T}$ and $P_{G}$. These small differences have implications on the angular distribution of vegetation indices calculated from the two bands and deserve further investigation.

Comparisons of the model results were made with measurements of a boreal black spruce forest [9]. Fig. 14(a) and 14(b) show the calculated and measured reflectance in the red band for two different $\theta_{s}$ values, $40^{\circ}$ and $55^{\circ}$, in the principal plane. At $\theta_{s}=40^{\circ}$, the model is overestimating the reflectance on the forwardcattering side. This is mainly 

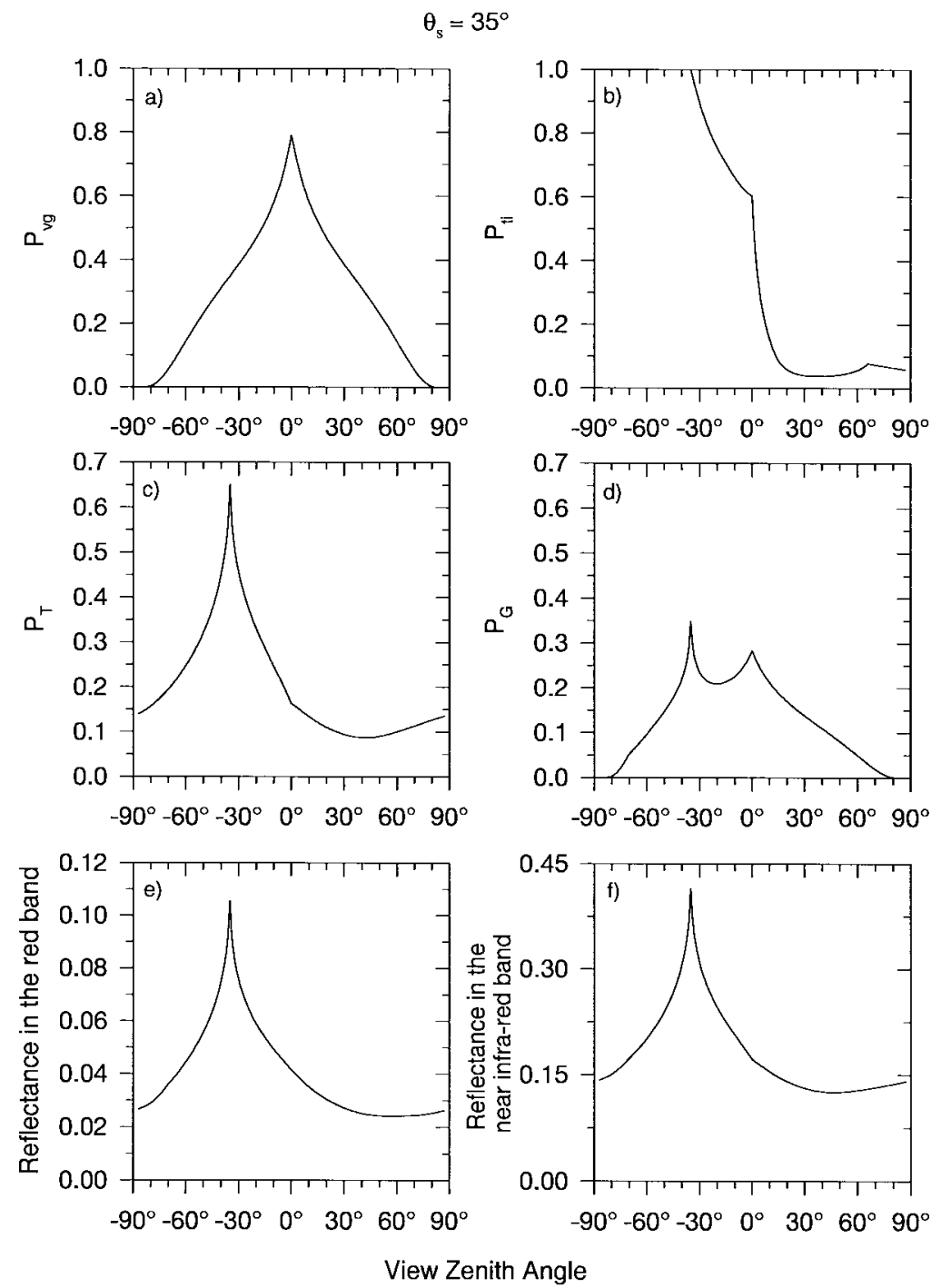

Fig. 13. Different components of the reflectance in the princpal plane at a solar zenith angle of $30^{\circ}$.

due to the constant reflectivities used for the shaded foliage and ground. The model uses constant values for $F_{d t}$ and $F_{d g}$ in (70) and (71) for the fraction of diffuse radiation in the total incident solar irradiance. As the fraction changes during the course of the day, these constant values better represent the average daily conditions. Near solar noon when the sun is high, the diffuse fraction is smaller than the daily average and the reflectivities for the shaded components are also correspondingly smaller than the daily average, resulting in the discrepancies between the modeled and observed values at $\theta_{s}=40^{\circ}$. The comparisons suggest that the model will benefit from accurate separation of the diffuse and direct solar radiation. The model shows sharp spikes at the hotspot in comparison with the measurements. The gentle variation in the measurements may be a result of the low angular resolution $\left(15^{\circ}\right)$ which effectively produced window-averaged results. The effect of the averaging is to dampen and broaden the peak at the hotspot. For $\theta_{s}=40^{\circ}$ and other small $\theta_{s}$ values not shown here, the width of the hotspot is well modeled. The model does not perform as well at the larger solar zenith angles like Fig. 14(b) for $\theta_{s}=55^{\circ}$. This model deficiency may have resulted from the simplified geometry used to represent the tree crowns. The model geometry is such that, all foliage is confined within the cone or cylinder, while in reality branches extend much further than the mean radius and intercept more radiation than the model prediction. One way to handle the problem is to increase the mean radius of the tree crowns, but in that case the branch structure needs to be more vigorously described to allow more gaps within the tree crown. This suggests that geometric-optical models are still approximations to reality and accurate simulation of BRD requires accurate descriptions of the canopy architecture at all scales. Fig. 14(c) shows a comparison between the model and measurements for the reflectance in the near-infrared band at $\theta_{s}=40^{\circ}$. The model is able to simulate the measurements closely. In Fig. 14(d) where $\theta_{s}=60^{\circ}$, the model performs well except for the largest $\theta_{v}$, especially on the backscattering side, indicating the effect of nonuniform distribution of multiple scattering on BRD which is not considered in the present study.

Fig. 15 shows hemispheric distributions of reflectance in the red band at four solar zenith angles. The hotspot varies in size, being generally larger when the sun is higher in the 


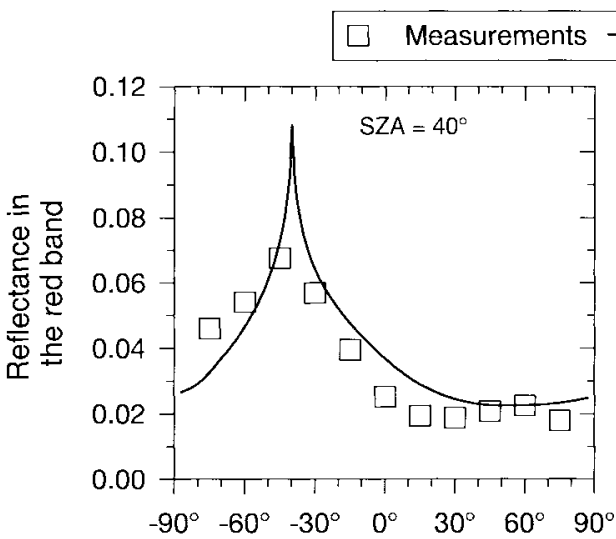

(a)

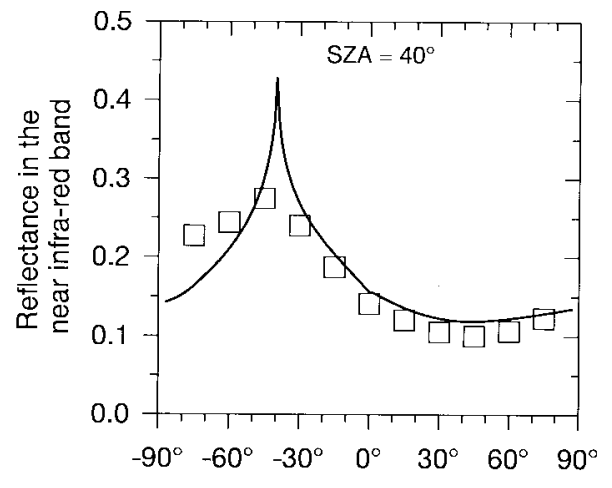

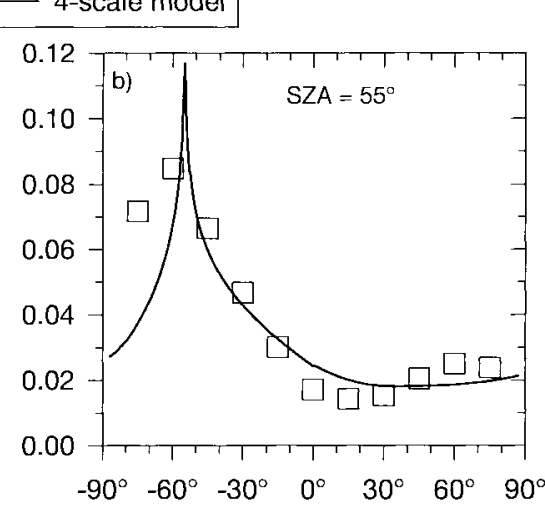

(b)

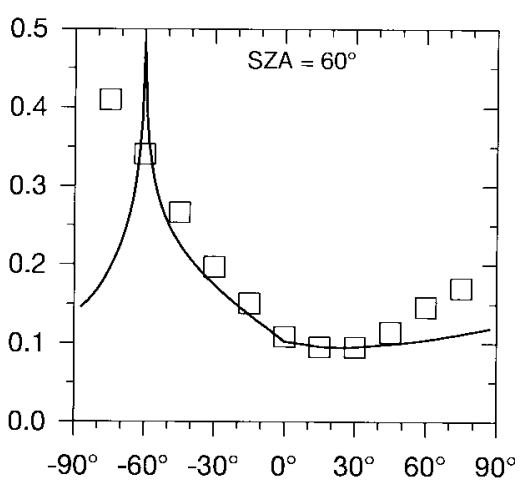

View Zenith Angle

(c)

(d)

Fig. 14. Comparisons of measured and modeled BRD in the principal plane for a black spruce in the red band for (a) $\theta_{s}=40^{\circ}$, and (b) $55^{\circ}$, and in the near infrared band for (c) $\theta_{s}=40^{\circ}$, and (d) $\theta_{s}=60^{\circ}$. The measurements were made by Deering et al. [9].

sky. The model does not show much of the usual bowl-shape because of the high LAI and the fixed diffuse fractions used in the model. Some of the bowl-shape can be seen in Fig. 15(c) and (d). Fig. 16 shows the corresponding distributions in the NIR band. The distributions are similar to those in Fig. 15 but the bowl-shape is more pronounced for $\theta_{s}=60^{\circ}$ and $75^{\circ}$. The increase in reflectance at large $\theta_{v}$ values, where the ground is hidden under the tree crowns is due in part to the larger reflectivity of the shaded foliage as compared to the shaded ground A singularity is seen at nadir for the large solar zenith angles, i.e., Fig. 16(d) because of the large shaded ground components viewed vertically.

\section{DISCUSSION}

The four-scale model presented here was developed to investigate the effect of canopy architecture at different scales on the bidirectional distribution. Since we have incorporated mathematics descriptions of canopy architecture at scales larger and smaller than the tree crown, it becomes a fuzzy geometric-optical model in the sense that the clearly defined canopy geometry, such as that of Li and Strahler's models, is disintegrated and defined probalistically. The inclusion of canopy architecture at the various scales in a geometric-optical model may be considered as an influx of negative entropy which increases the orderliness of the system under investiga- tion and is expected to approximate more closely natural living organizms. We believe that such a model contains more flexibility to adapt to different plant canopies than previous models.

Much mathematical complexity arises, though still tractable, when we treat the tree crown surface as a complex surface within which mutual shadows and the hotspot also occur. Through our numerical simulation, we believe that the subcanopy structures have profound effects on the directional reflection behavior of the canopy and deserve such attention. Fig. 17 shows the sensitivity of the model to the foliage density within tree crowns. With the fixed stand density and tree crown shape and dimensions (Table I), the foliage density increases proportionally with LAI. For these simulations, we kept the multiscattering factors constant, and realized that some inaccuracies occurred in the model results because larger LAI should induce more multiscattering in the canopy. Both the red and NIR reflectances increase with decreasing LAI in the forward scattering direction due to the increased probabilities of observing sunlit foliage from the shaded side through sparse tree crowns. The hotspot is smaller for a sparser canopy (lower LAI) because of the increased probability of observing the ground, which has a lower reflectivity than the foliage. These model results support the findings of Soffer [32] that the measured reflectance on the shaded side of jack pine crowns is considerably larger than the predictions of a $\mathrm{Li}$ and 


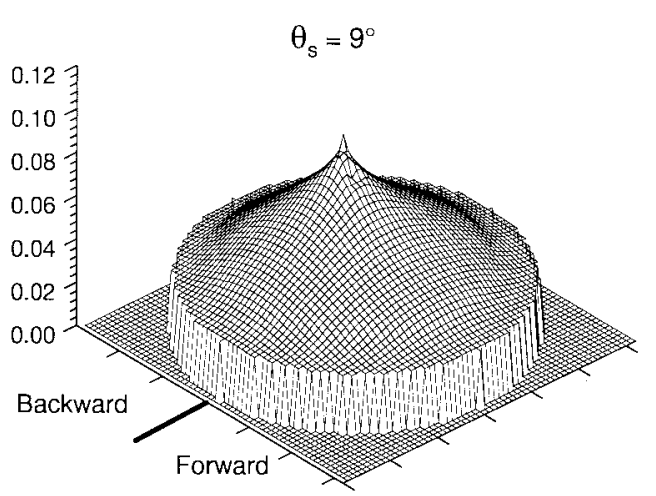

(a)

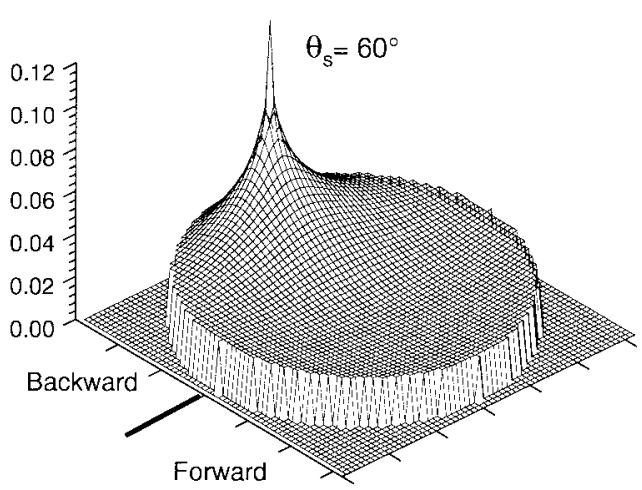

(c)

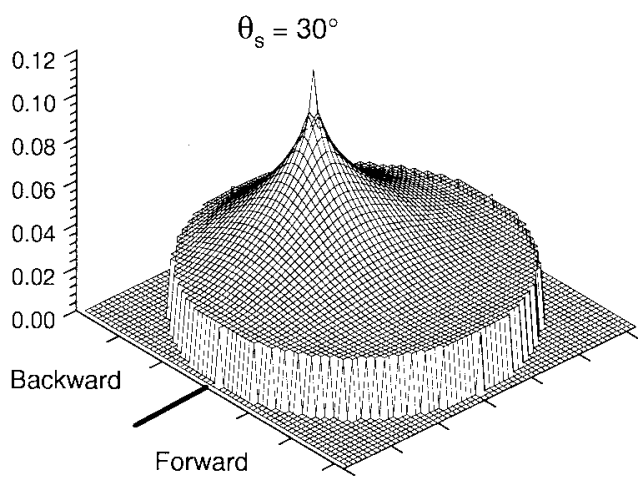

(b)

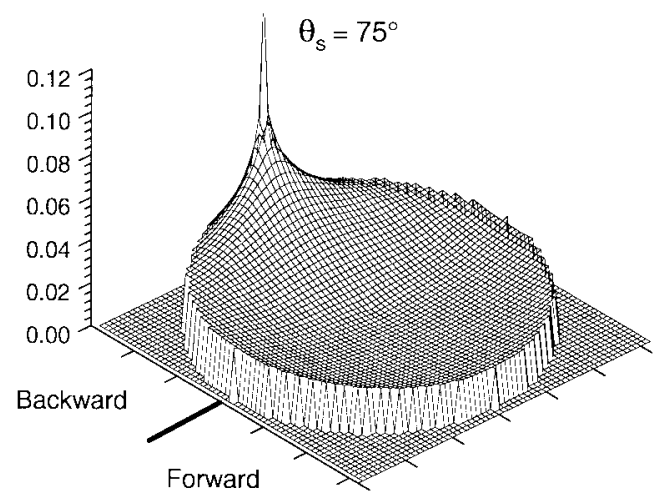

(d)

Fig. 15. Modeled hemispherical reflectance distributions in the red band at four solar zenith angles $\left(\theta_{s}\right)$.

Strahler model. Black spruce crowns are generally denser and the shaded side appears darker than the jack pine. Our model is able to simulate such differences.

The assumption of random tree distribution is removed at the expense of lengthy mathematical descriptions using the combined Neyman and binomial distributions. Apparently, such effort has not been very rewarding with regard to its impact on the final BRD results for the stand investigated. Fig. 18(a) shows that the Neyman grouping increases the reflectance in the red band only on the forward scattering side near the vertical direction. The increased openness of the forest due to the Neyman grouping is the main cause of the increase in the modeled reflectance. The Neyman grouping decreases the width of the hotspot because a larger $m_{2}$ gives a smaller $\Omega_{t}$ which affects the hotspot kernel for the ground. At large view angles, a larger Neyman grouping gives a smaller reflectance because the contribution of the ground to the reflectance becomes very small and the tree crown properties dominate at these angles. The change of the hotspot width is more important in the NIR than the red band because the Neyman grouping affects the proportion of the ground illuminated $\left(P_{G}\right)$. Our present simulation is limited to small quadrats with small Neyman groupings. More research is needed to determine the grouping effect at larger scales (large quadrats with large groupings).

Sub-canopy architecture is not only important in investigating the BRD of vegetated surfaces but also critical in the inversion of the model to obtain biophysical parameters such as leaf area index. Clumping of needles within shoots, for example, affects the radiation interception in the plant canopy and hence vegetation indices derived from the reflectances in red and near-infrared bands. Grouping of shoots in branches also has similar effects. Accurate estimation of LAI from the model requires appropriate descriptions of architecture at these sub-canopy levels.

In this paper, the effect of multiple scattering on BRD is not investigated. Our attention is first given to canopy architecture because the first order scattering is usually much larger than the sum of multiple scattering and the angular distribution of the multiply scattered radiances is usually considered to be isotropic [31]. However, at NIR wavelengths, the multiple scattering effect is larger than that at visible wavelengths and the isotropic assumption may lead to some inaccuracies in the BRD results. Such subtle differences may have significant effects on the simulated vegetation indices using reflectance factors for these two bands. We hold the believe that in modeling the multiple scattering, the canopy architecture is foremost important because it dictates the direction of first order scattering and the probability of observing the reflecting surfaces at different steps of the scattering sequence. The approaches of Goel et al. [14] and Strahler and Jupp [33] in the description of plant canopy architecture have such merits, but forest canopies are much more complex than what have been described. The model presented here provides a framework in which the effect of canopy architecture at various scales on BRD can be systematically investigated. 


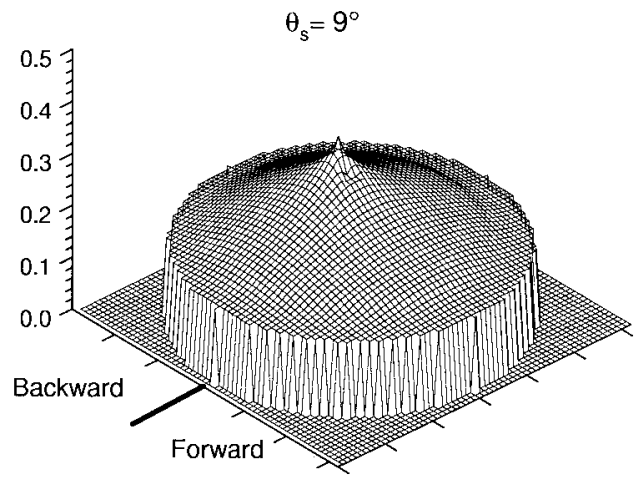

(a)

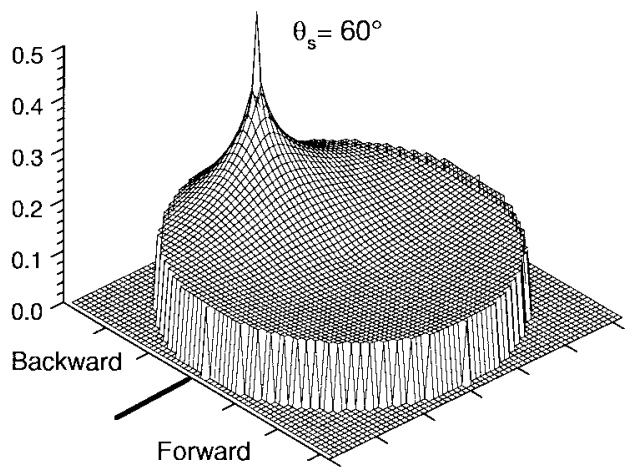

(c)

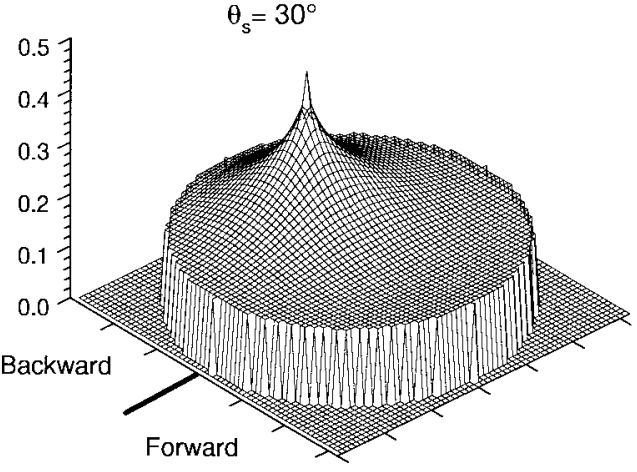

(b)

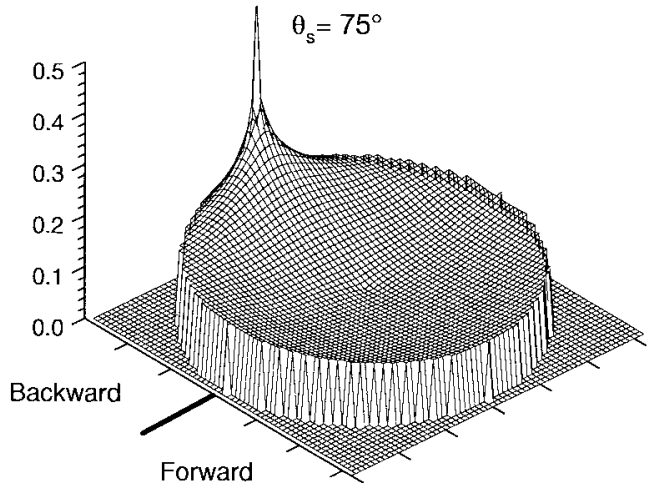

(d)

Fig. 16. Modeled hemispherical reflectance distributions in the NIR band at four solar zenith angles $(\theta)$.

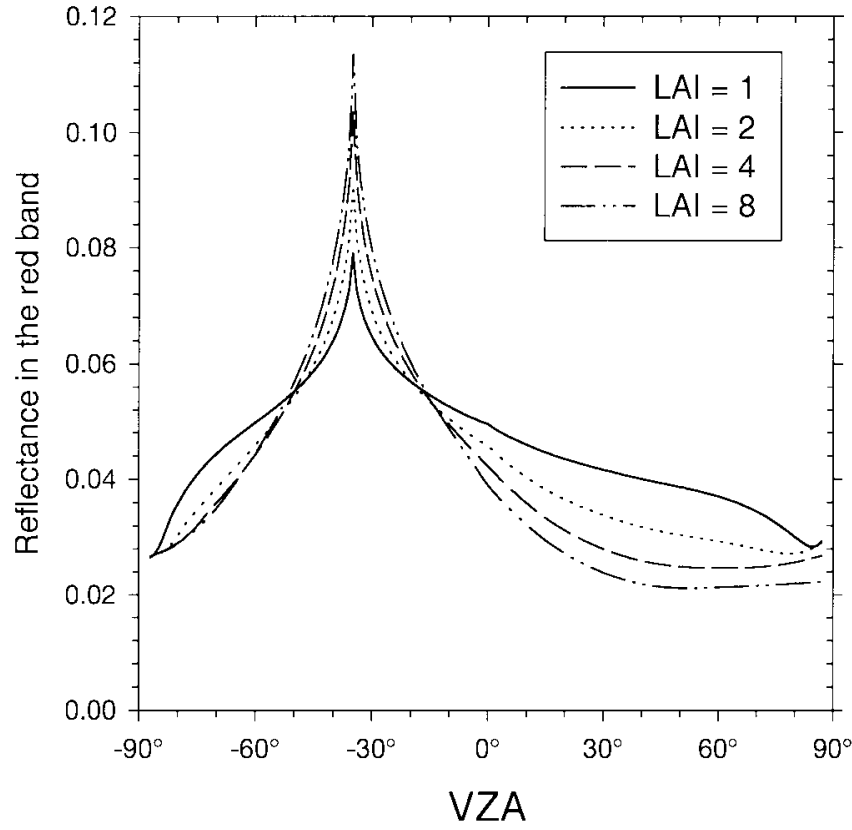

(a)

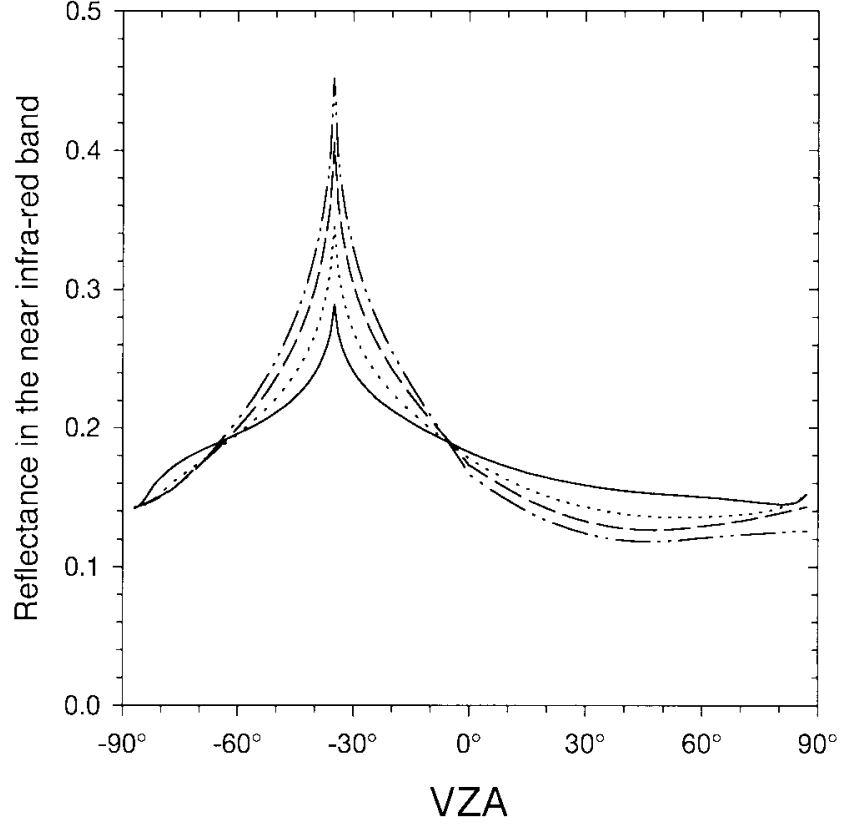

(b)

Fig. 17. Effects of foilage density in tree crowns determined by LAI on the BRD in the principal plane, (a) red band, (b) near-infrared band. $\theta_{s}=$ $35^{\circ}$. In the simulation the tree density is fixed at 4000 stems/ha.

\section{CONCLUSION}

In contrast to the turbid-medium type of models suitable for short vegetation canopies without distinct foliage structures, geometric-optical models are more appropriate for forest canopies which are usually well organized at various scales. Compared with previous two-scale geometric-optical models, which describe trees as randomly-distributed discrete objects containing turbid media, the four-scale model presented in this 


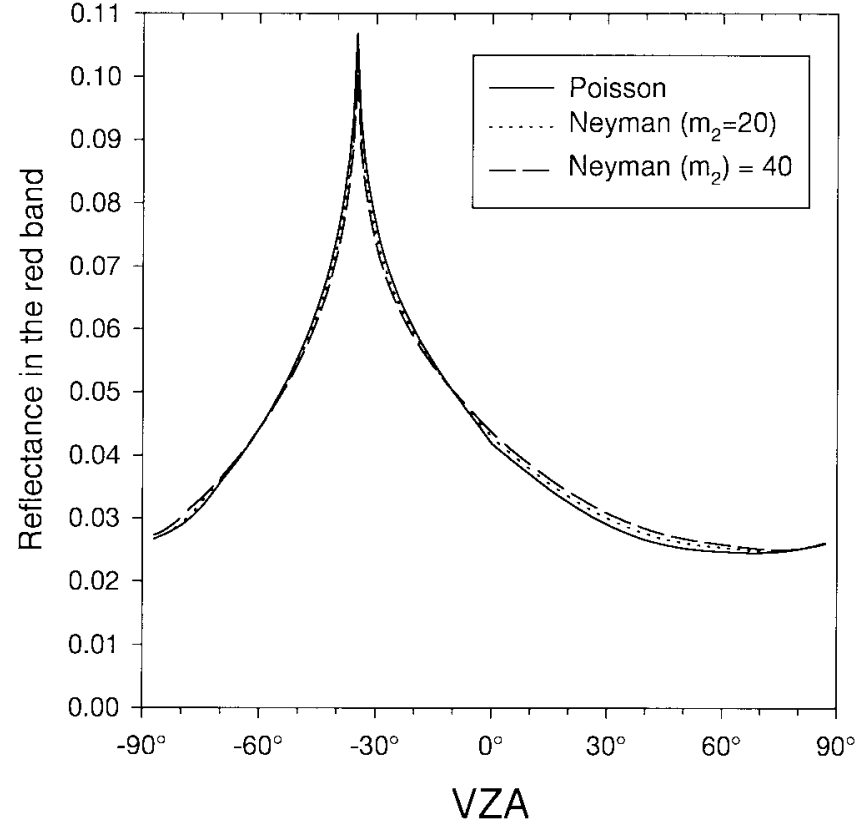

(a)

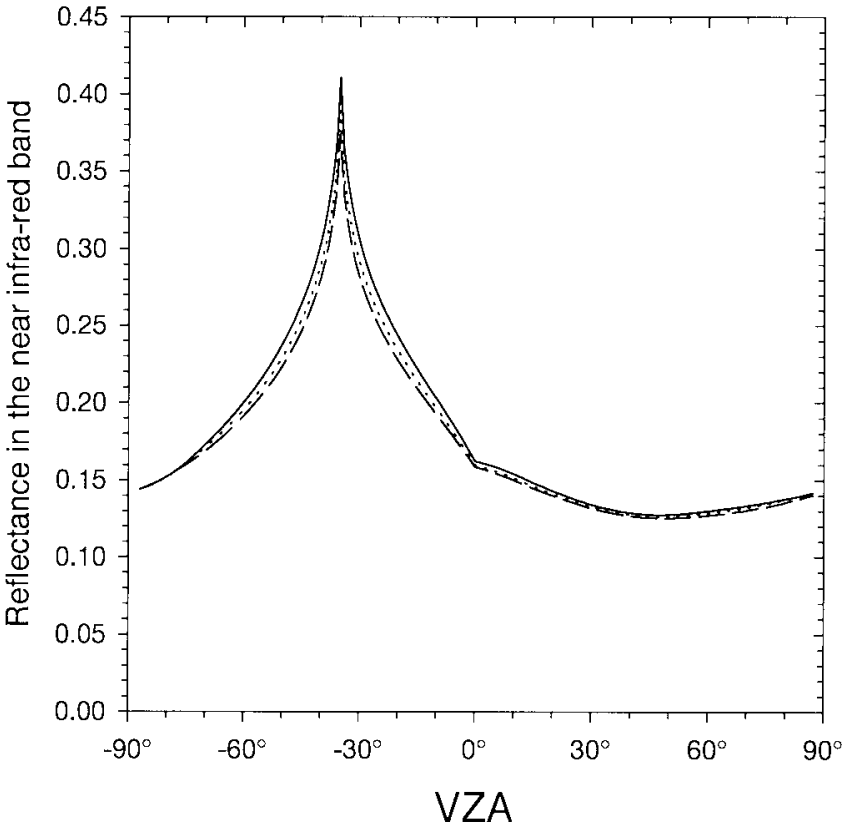

(b)

Fig. 18. Effects of Neyman grouping on the BRD in the principal plane: (a) red band and (b) infra-red band. $\theta_{s}=35^{\circ}$.

paper includes the effects of two additional scales of canopy architecture: tree distribution pattern and foliage distribution pattern within trees. The four-scale model simulates closely the measurements of tree distribution, canopy gap fraction and the bidirectional reflectance. It is shown in model simulation that the architecture within tree crowns has profound effects on the bidirectional reflectance distribution (BRD). Tree distribution patterns have small but significant effects on BRD for the stand investigated and may have larger effects for more patchy stands.

In our model, a tree crown consists of conical and cylindrical parts. An effective mathematical scheme is devised to estimate the mutual shadowing effect between tree crowns and among foliage elements within tree crowns. The canopy gap size distributions between and within tree crowns are used to describe the hotspot size and shape. The description, for the first time, closely relates the bidirectional reflection behavior to canopy attributes.

\section{APPENDIX \\ CONE GEOMETRY}

The computation of the proportion of illuminated area on a cone is done using simple geometry. The area of the cone projected to a viewer can be separated into two parts: an ellipse and a triangle. The nine schematic representations in Fig. 19 show the typical shaded areas viewed on the cone. The origin of the coordinates is always the centre of the ellipse and the $\mathrm{x}$-coordinate of each point stays the same. The ellipse is expressed by

$$
\frac{x^{2}}{x_{A}^{2}}+\frac{y^{2}}{r^{2}}=1
$$

where $x_{A}=r \cos \left(\theta_{v}\right) ;\left(x_{A}, y_{A}\right)$ is point $\mathrm{A}$; and $y_{A}=0 . r$ is the base radius of the cone. For $\theta_{v} \leq \alpha$ the cone area seen is

$$
t_{a c}=\pi \cdot r \cdot x_{A}=\pi r^{2} \cos \left(\theta_{v}\right) .
$$

When $\theta_{v}>\alpha$, the point $B$ is outside the ellipse. This new area (called $t_{\text {act }}$ in the paper) can be easily computed by integrating twice from the ellipse to the segment $\overline{B D}$ from 0 to $y_{D}$ :

$$
\begin{aligned}
t_{a c}= & \pi r^{2} \cos \left(\theta_{v}\right) \\
& +2 \int_{0}^{y_{D}}\left[\frac{x_{A}}{r} \sqrt{r^{2}-y^{2}}-\frac{y-b_{B D}}{m_{B D}}\right] d y
\end{aligned}
$$

where $b_{B D}$ and $m_{B D}$ are the intercept and the slope of the $\overline{B D}$ segment. $\left(x_{B}, y_{B}\right)$ denotes point $\mathrm{B}$, which is the projected tip of the cone $\left(y_{B}=0\right) \cdot\left(x_{D}, y_{D}\right)$ is the intercept between the triangle and the ellipse. The integral in (75) has an analytical solution.

When the solar zenith angle $\theta_{s}$ is larger than the apex angle $\alpha$, self-shadowing on the cone occurs. At nadir, the illuminated area is expressed by

$$
t_{i c}=\left[\frac{\pi}{2}+\gamma\right] r^{2}
$$

where $\gamma=\sin ^{-1}\left[\tan (\alpha) / \tan \left(\theta_{s}\right)\right]$ [19]. This is valid for all azimuth angles $\phi$. On the backscattering side of the principal plane [Fig. 19(a)], the shadow can be seen while $\theta_{v}<\theta_{s}$. For $\theta_{v} \leq \alpha$ we have

$$
t_{i c}=y_{E} \cdot\left(x_{E}-x_{B}\right)+r x_{A} \cdot[\sin (\gamma) \cos (\gamma)-\gamma+\pi / 2]
$$

where $\left(x_{E}, y_{E}\right)$ denotes point $E \cdot x_{E}=x_{A} \cdot \sin (\gamma+\phi)$ and $y_{E}=r \cdot \cos (\gamma+\phi)$ As $\theta_{v}$ increases, the triangular part appears to the viewer. Two shadowed areas can be seen on the triangle part. Outside the principal plane, the symmetry does not exist, but the same equations can still be used. The third plate of Fig. 19(b) shows such a case. The computation of the 

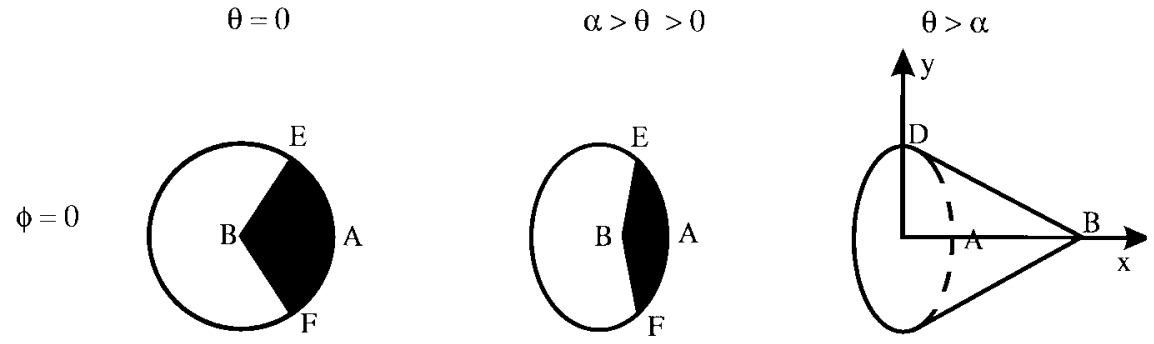

(a)
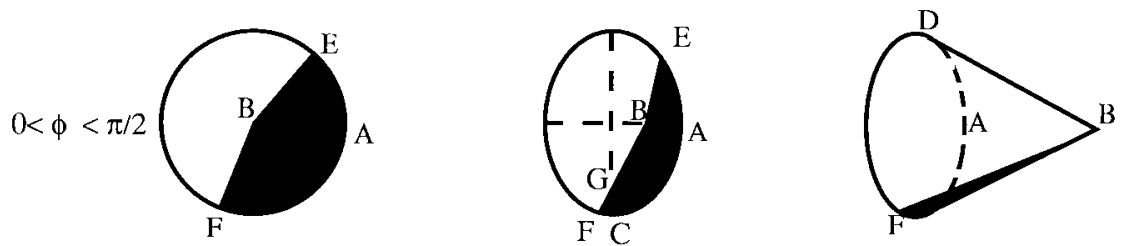

(b)
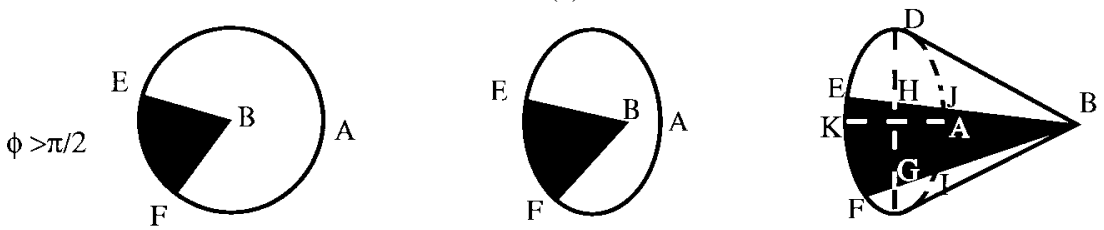

(c)

Fig. 19. Examples of illuminated cone surface area $\left(t_{i c}\right)$ at different view angles.

shaded area is an integration of areas inside the ellipse which is separated into four parts to facilitate the selection of integral limits. Fig. 19(b) for $\alpha>\theta_{v}>0$ we have the shadowed area in three parts of the ellipse, it is calculated as follow:

$$
\begin{aligned}
\int_{0}^{y_{E}} & {\left[\frac{y-b_{B E}}{m_{B E}}-\frac{x_{a}}{r} \sqrt{r^{2}-y^{2}}\right] d y } \\
& +\frac{\pi}{4} \cdot r^{2} \cos \left(\theta_{v}\right)-\frac{\left|x_{B} \cdot b_{B F}\right|}{2} \\
& +\int_{0}^{x_{F}}\left[m_{B F} x+b_{B F}-\frac{r}{x_{a}} \sqrt{x_{A}^{2}-x^{2}}\right] d y .
\end{aligned}
$$

The first term is for the area formed by the points $E, B$, and $A$, denoted $E B A$, the second and third are for the area $A B G C$ and $G F C$, respectively. The point $G$ is the intercept of $\overline{B F}$ on $y$. The area illuminated $\left(t_{i c}\right)$ is found by substracting (78) from (75).

In (78), $m_{B E}$ and $b_{B E}$ are the slope and intercept of $\overline{B E}$ on y defined by the equation of the curve that has the 2 points $B$ and $E . m_{B F}$ and $b_{B F}$ have similar definitions. $y_{E}$ and $y_{F}$ do not depend on $\theta_{v}$. Once they are found at nadir, their values can be apply to other view angle at any given $\phi$. When $\theta_{v}>\alpha$, the amount of shadowed surface in the triangle is calculated as follows: the integration is like the one in (75). We divide the triangle into two parts separated by the $\mathrm{x}$-axis. The areas of the two triangles are:

$$
\int_{0}^{y_{I}}\left[\frac{x_{A}}{r} \sqrt{r^{2}-y^{2}}-\frac{y-b_{B E}}{m_{B E}}\right] d y
$$

and

$$
\int_{0}^{y_{J}}\left[\frac{x_{A}}{r} \sqrt{r^{2}-y^{2}}-\frac{y-b_{B F}}{m_{B F}}\right] d y
$$

The shadow on the ellipse is, as above, calculated by separating the ellipse into four parts: $H O A J, G O A I, F G O K$, and $E H O K$.

\section{ACKNOWLEDGMENT}

The authors are indebted to Dr. D. Deering and Dr. T. Eck for the permission to use a sample of PARABOLA data. They thank Dr. J. Cihlar for his support and stimulating discussions in the development of the model. The paper was internally reviewed by Dr. R. Gauthier and Dr. P. Teillet before submission. They also thank L. Moreau for technical discussions. The model in $\mathrm{C}$ code is available on request.

\section{REFERENCES}

[1] C. C. Borel, A. W. Gerstl, and B. Powers, "The radiosity method in optical remote sensing of structured 3-D surfaces," Remote Sens. Environ., vol. 36, pp. 13-44, 1991.

[2] J. M. Chen, "Canopy architecture and remote sensing of the fraction of photosynthetically active radiation absorbed by boreal conifer forests," IEEE Trans. Geosci. Remote Sensing, vol. 34, pp. 1353-1368, 1996.

[3] J. M. Chen and T. A. Black, "Measuring leaf area index of plant canopies with branch architecture," Agric. Forest Meterol., vol. 57, pp. 1-12, 1991.

[4] _ "Defining Leaf Area Index for nonflat leaves," Plant Cell Environ., vol. 15, pp. 421-429, 1992.

[5] _ "Foliage area and architecture of plant canopies from sunfleck size distributions," Agric. Forest Meteorol., vol. 60, pp. 249-266, 1992.

[6] J. M. Chen and J. Cihlar, "Plant canopy gap size analysis theory for improving optical measurements of leaf area index," Appl. Opt., vol. 34, pp. 6211-6222, 1995.

[7] _ "Quantifying the effect of canopy architecture on optical measurements of leaf area index using two gap size analysis methods," IEEE Trans. Geosci. Remote Sensing, vol. 33, pp. 777-787, 1995.

[8] J. Cihlar, D. Manak, and N. Voisin, "AVHRR bidirectional reflectance effect and compositing," Remote Sens. Environ., vol. 48, pp. 77-88, 1994. 
[9] D. W. Deering, S. P. Ahmad, and T. F. Eck, "Temporal attributes of the bidirectional reflectance for three boreal forest canopies," Proc. 1995 IEEE Geosci. Remote Sensing Symp., pp. 1239-1241.

[10] D. W. Deering, E. M. Middleton, T. F. Eck, and B. P. Banerjee, "Reflectance anisotropy for a spruce-hemlock forest canopy," Remote Sens. Environ., vol. 47, pp. 242-260, 1994.

[11] J. Franklin, J. Michaelsen, and A. H. Strahler, "Spatial analysis of density dependent pattern in coniferous forest stands," Vegetatio, vol. 64, pp. 29-36, 1985.

[12] W. Gao, "A simple bidirectional-reflectance model applied to a tallgrass canopy," Remote Sens. Environ., vol. 45, pp. 209-223, 1992.

[13] A. Getis and B. Boots, Models of Spatial Processes, An Approach to the Study of Point, Line and Area Patterns. Cambridge, U.K.: Cambridge Univ. Press, 1978.

[14] N. S. Goel and W. Qin, "Influences of canopy architecture on relationships between various vegetation indices and LAI and FPAR: A computer simulation," Remote Sens. Rev., vol. 10, pp. 309-347, 1994.

[15] N. S. Goel, I. Rozehnal, and R. L. Thompson, "A computer graphics based model for scattering from objects of arbitrary shapes in the optical region," Remote Sens. Environ., vol. 36, pp. 73-104, 1991.

[16] B. Hapke, D. DiMucci, R. Nelson, and W. Smythe, "The cause of the hot spot in vegetation canopies and soil: Shadow-hiding versus coherent backscatter," Remote Sens. Environ., vol. 58, pp. 63-68, 1996.

[17] D. L. B. Jupp and A. H. Strahler, "A hotspot model for leaf canopies," Remote Sens. Environ., vol. 38, pp. 193-210, 1991.

[18] A. Kuusk, "The hotspot effect of a uniform vegetation cover," Sov. J. Remote Sens., vol. 3, pp. 645-658, 1985.

[19] X. Li and A. H. Strahler, "Geometric-optical modeling of a conifer forest canopy," IEEE Trans. Geosci. Remote Sensing, vol. GE-23, pp. 705-721, 1985.

[20] _ _ "Geometric-optical bidirectional reflectance modeling of a coniferous forest canopy," IEEE Trans. Geosci. Remote Sensing, vol. GE-24, pp. 906-919, 1986

[21] _ "Modeling the gap probability of a dicontinous vegetation canopy," IEEE Trans. Geosci. Remote Sensing, vol. 26, pp. 161-169, 1988.

[22] _ "Geometric-optical bidirectional reflectance modeling of the discrete crown vegetation canopy: Effect of crown shape and mutal shadowing," IEEE Trans. Geosci. Remote Sensing, vol. 30, pp. 276-291, 1992.

[23] X. Li, A. H. Strahler, and C. E. Woodcock, "A hybrid geometricoptical-radiative transfert approach for modeling albedo and directional reflectance of discontinuous canopies," IEEE Trans. Geosci. Remote Sensing, vol. 33, pp. 466-480, 1995.

[24] E. M. Middleton, D. W. Deering, and S. P. Ahmad, "Surface anisotropy and hemispheric reflectance for a semiarid ecosystem," Remote Sens. Environ., vol. 23, pp. 193-212, 1987.

[25] E. E. Miller and J. M. Norman, "A sunfleck theory for plant canopies. I. lengths of sunlit segment along a transect," Agron. J, vol. 63, pp. 735-738, 1971.

[26] R. B. Myneni and J. Ross, Eds., Photon-Vegetation Interactions. Berlin, Germany: Springer-Verlag, 1991.

[27] J. Neyman, "On a new class of 'contagious' distributions, applicable in entomology and bacteriology," Ann. Math. Statist., vol. 10, pp. 35-57, 1939.

[28] T. Nilson, "A theoretical analysis of the frequency of gaps in plant stands," Agric. Meteorol., vol. 8, pp. 25-38, 1971.

[29] T. Nilson and U. Peterson, "A forest canopy reflectance model and a test case," Remote Sens. Environ., vol. 37, pp. 131-142, 1991.
[30] J. Otterman and T. W. Brakke, "Dense canopy albedo as a function of illumination direction: Dependance on structure and leaf transmitance," Theor. Appl. Climat., vol. 43, pp. 3-16, 1991.

[31] J.-L. Roujean, "A bidirectional reflectance model of the earth's surface for the correction of remote sensing data," J. Geophys. Res., vol. 43, pp. 3-16, 1991

[32] R. Soffer, "Bidirectional reflectance factors of an open tree canopy by laboratory simulation," M.S. thesis, York University, North York, Ont., Canada, 1995.

[33] A. H. Strahler and D. L. B. Jupp, "Modeling bidirectional reflectance of forests and woodlands using boolean models and geometric optics," Remote Sens. Environ., vol. 34, pp. 153-166, 1990.

[34] W. Verhoef, "Light scattering by leaf layers with appliction to canopy reflectance modeling: The Sail model," Remote Sens. Environ., vol. 16, pp. 125-141, 1984

[35] M. Verstraete, "Radiation transfer in plant canopies: scattering of solar radiation and canopy reflectance," J. Geophys. Res., vol. 93, pp. 9483-9494, 1988.

[36] H. P. White, J. R. Miller, J. M. Chen, D. R. Peddle, and G. McDermid, "Seasonal change in mean understory reflectance for boreal sites: Preliminary results," Proc. 17th Can. Symp. Remote Sensing., 1995.

[37] A. Wu, Z. Li, and J. Cihlar, "Effect of land cover type and greenness on advanced very high resolution radiometer reflectance: Analysis and removal," J. Geophys. Res., vol. 100, pp. 9179-9192, 1995.

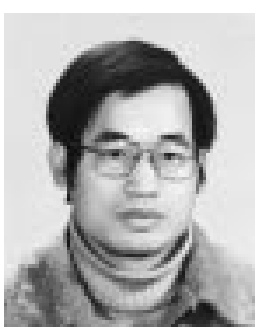

Jing M. Chen received the B.Sc. degree in 1982 from Nanjing Institute of Meteorology, China, an the Ph.D. degree in 1986 from University of Reading, Reading, U.K.

He is currently a Research Scientist at the Canada Centre for Remote Sensing (CCRS), Ottawa, Ont., and an Adjunct Professor at the University of Ottawa and York University, North York, Ont. His main research interests have been in turbulent and radiative transfer processes associated with plan canopies. He is currently engaged in research on applications of optical and microwave remote sensing techniques to boreal ecosystems. The research topics include radiation modeling, biophysical parameter retrieval, and modeling net primary productivity and carbon cycle. He has been a principal investigator in Boreal Ecosystem-Atmosphere Study, VEGETATION/SPOT applications and other projects.

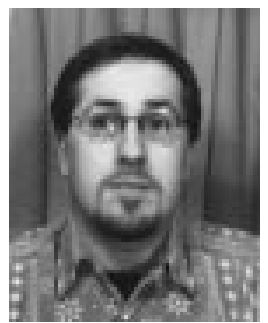

Sylvain G. Leblanc received the B.Sc. degree in physics from the University of Montréal, Montréal, P.Q., Canada, in 1992, and the M.Sc. degree in atmospheric and oceanic sciences from McGill University, Montréal, in 1994, on remote sensing of turbulence.

$\mathrm{He}$ is currently with Intermap Technologies, $\mathrm{Ne}$ pean, Ont., Canada, and works as a Research Associate at the Canada Centre for Remote Sensing (CCRS), Ottawa, Ont. His current research includes remote sensing and modeling of biophysical prop- 